\title{
Pour une géo-archéologie du patrimoine : pierres, carrières et constructions en Bretagne
}

sixième partie : les granites cadomiens du batholite mancellien

For a Geo-Archaelogy of Heritage: stones, quarries and buildings in Brittany

Für eine Geoarchäologie des kulturellen Erbes: Gesteine, Steinbrüche und

Gebäude in der Bretagne.

Para una geo-arqueología del patrimonio: piedras, yacimientos y construcciones en Bretaña.

\section{Louis Chauris}

\section{(2) OpenEdition}

\section{Journals}

Édition électronique

URL : http://journals.openedition.org/rao/2755

DOI : $10.4000 /$ rao. 2755

ISBN : 978-2-7535-4053-8

ISSN : 1775-3732

\section{Éditeur}

Presses universitaires de Rennes

\section{Édition imprimée}

Date de publication : 15 décembre 2014

Pagination : 409-430

ISBN : 978-2-7535-4051-4

ISSN : 0767-709X

\section{Référence électronique}

Louis Chauris, «Pour une géo-archéologie du patrimoine : pierres, carrières et constructions en Bretagne », Revue archéologique de l'Ouest [En ligne], 31 | 2014, mis en ligne le 15 décembre 2016, consulté le 03 décembre 2020. URL : http://journals.openedition.org/rao/2755 ; DOI : https://doi.org/ $10.4000 /$ rao. 2755 


\title{
Pour une géo-archéologie du patrimoine : pierres, carrières et constructions en Bretagne
}

\author{
For a Geo-Archaelogy of Heritage: Stones, Quarries and Buildings in Brittany
}

\author{
Louis Chauris*
}

\section{Sixième partie : les granites cadomiens du batholite mancellien ${ }^{1}$ Part six: Cadomian Granites of the Mancellian Batholith}

\begin{abstract}
Résumé : L'est de la Bretagne n'est concerné que par les plutons les plus occidentaux du grand batholite granitique mancellien mis en place dans le nord-est du Massif armoricain à la fin du Précambrien. Ils présentent fréquemment un certain « air de famille ». Les uns (Mont-Dol, SaintBroladre, Dingé, Bécherel) n’ont eu essentiellement qu'une utilisation locale et sont aujourd'hui abandonnés. Deux autres, toujours exploités, ont une diffusion nationale (Louvigné), voire internationale (Lanhélin), justifiant de plus amples développements.
\end{abstract}

\begin{abstract}
East of Brittany is concerned only by the most occidental plutons of the large Mancellian granitic batholith formed in the North-East of the Armorican massif by the end of Precambrian times. These plutons often have a "family likeness". Many of them (Mont-Dol, Saint-Broladre, Dingé, Bécherel) have had a mainly local use and are now abandoned. Two others are still exploited, with a national diffusion (Louvigné) or even an international one (Lanhélin), which justifies a more detailed presentation.
\end{abstract}

Mots clés : Mancellia, granites, extractions, utilisations, Louvigné, Lanhélin.

Keywords: Mancellia, Granites, extraction, uses, Louvigné, Lanhélin.

\section{VUE D'ENSEMBLE SUR LES GRANITES DU BATHOLITHE MANCELLIEN}

La dénomination "Mancellia ", créée par P. Pruvost (1949), se rapporte aux terrains situés dans le nord-est du Massif armoricain entre le bassin de Laval au sud et la « zone bocaine " au nord. En Ille-et-Vilaine, seuls affleurent les plutons les plus occidentaux de l'immense batholite mancellien, à savoir Louvigné-du-Désert (qui se poursuit aussi dans les départements de la Manche et de la Mayenne), Lanhélin,
Saint-Broladre, Dingé, Bécherel... ainsi que quelques petits pointements (Mont-Dol...). L'âge de ces granites a été longtemps discuté. Ils ont d'abord été reconnus comme antérieurs au Cambrien par A. de Lapparent (1877), puis rapportés à la chaîne hercynienne (Vandernotte, 1913). Plus récemment à nouveau attribués à l'évolution tardi-cadomienne (Graindor, 1953; Chauris, 1956; Chauris et al., 1956); cette détermination est pleinement confirmée par les mesures géochronologiques (selon les auteurs, les âges oscillent entre 580 et 530 millions d'années). En un mot,

* Directeur de recherche au CNRS (e. r.) - 3 rue Goethe, 29200 BREST.

1. Cet article fait suite aux cinq déjà parus dans la RAO : $\mathrm{n}^{\circ} 26$ (2009), p. 259-283; nº 27 (2010), p. 171-207; n 28 (2011), p. 219-245; nº 29 (2012), p. 287-316; nº 30 (2013), p. 261-288. 
tous ces plutons sont aujourd'hui considérés comme faisant partie d'un gigantesque batholite fini-précambrien (Jonin, 1981).

Sous cette unité d'ensemble et un " air de famille » indéniable, les différents plutons du batholite mancellien laissent percer une certaine diversité (parfois même à l'intérieur d'un seul massif), allant des granodiorites aux leucogranites. Sur la carte, de forts contrastes apparaissent dans les contours et les dimensions des plutons. Le vaste massif de LouvignéGorron (70 km d'ouest en est) présente des limites irrégulièrement contournées, dues au moins en partie aux caprices liés à la profondeur atteinte par l'érosion. Inversement, Saint-Broladre, et dans une moindre mesure Dingé, dessinent une ellipse presque parfaite. Le petit pointement du Mont-Dol offre un contour piriforme; Bécherel étonne par son allongement démesuré par rapport à sa largeur (fig. 1).

Intrusifs au sein des schistes briovériens facilement érodables, les plutons du batholite offrent une nette tendance à apparaitre en relief. Le cas le plus frappant est sans conteste celui du Mont-Dol surgissant d'une soixantaine de mètres au-dessus des polders, mais il est loin d'être unique. SaintBroladre domine d'environ soixante-dix mètres la plaine septentrionale, mais dans ce cas, les cornéennes du contact, très indurées, ont joué un rôle morphologique indubitable en constituant comme un corset protecteur autour du granite; le long de sa bordure occidentale, le granite de Dingé, lui aussi, śélève à une quarantaine de mètres au-dessus des schistes briovériens. Il n'est pas jusqu'à la toponymie qui ne reflète l'impact du granite dans l'environnement. À l'échelle d'un pointement pris dans son ensemble, cette influence est évidente pour le Mont-Dol. Mais aussi dans le détail : la présence de boules, souvent énormes, parfois sculptées par l'érosion naturelle, a frappé depuis longtemps l'imagination populaire... où le diable apparaît comme un "intermédiaire » obligé.

Loriginalité pétrographique des granites du batholite mancellien se traduit par leur nuance bleutée, très accusée à Lanhélin mais encore sensible à Dingé, à Bécherel, à SaintBroladre et à Louvigné; toutefois, les faciès marginaux et les petits pointements à évolution leucogranitique n'offrent pas cette teinte. L'atout conféré par cette coloration dominante n'est pas sans promouvoir aujourd'hui la renommée lointaine du granite de Lanhélin.

\section{Le LeUCogranite DU MonT-Dol}

Dans le vaste domaine des sciences de la Terre, le MontDol doit sa célébrité au site paléolithique découvert à son pied par Simon Sirodot en 1872 (Monnier et Molines, 2003). Comme Tombelaine et le Mont-Saint-Michel (en Normandie et, par suite, en dehors de notre propos) et encore "insulaires ", le Mont-Dol a été longtemps un îlot, rattaché au domaine terrestre à l'époque post-glaciaire. Il constitue le plus étendu de ces trois pitons, avec une longueur de 750 mètres et une largeur maximale légèrement supérieure à 300 mètres. Il forme un pointement leucogranitique intrusif dans les formations schisto-gréseuses du Briovérien où il a développé un métamorphisme de contact. Les cornéennes ainsi formées affleurent sur le ver-

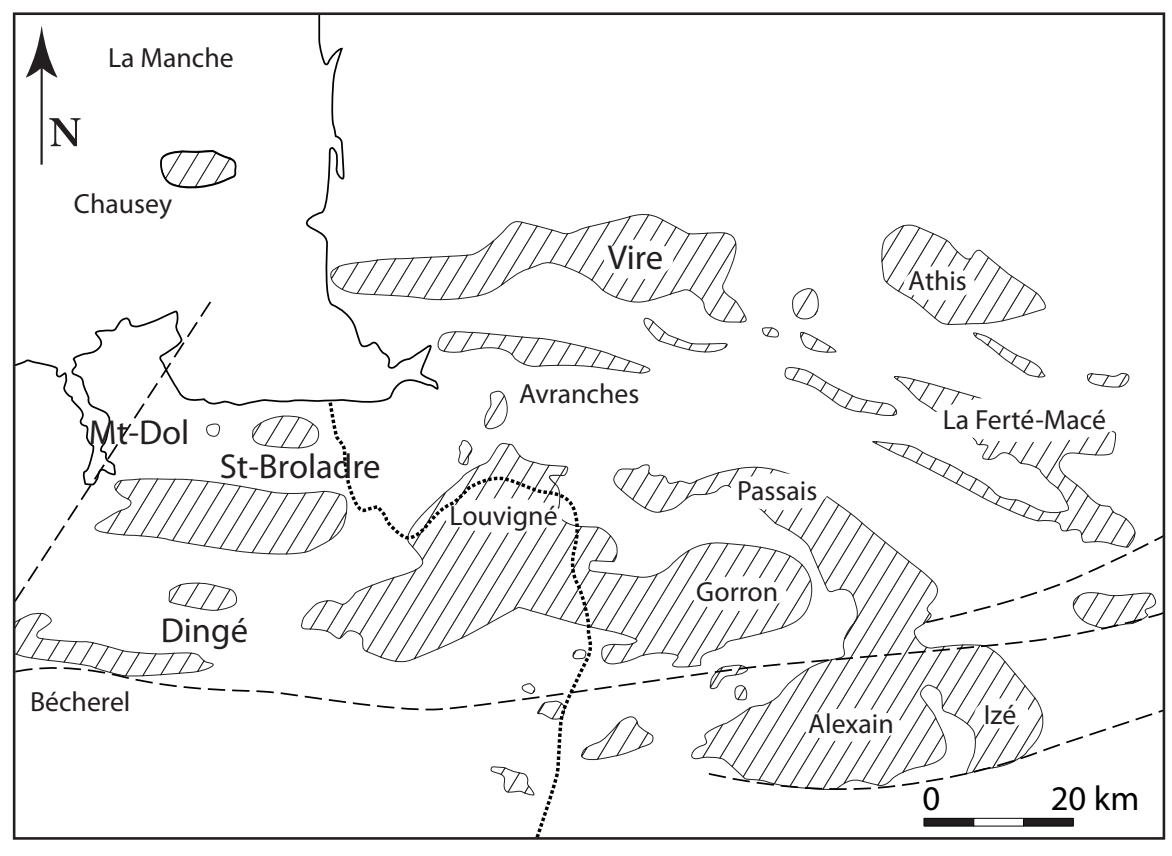

Figure 1 : Le batholite cadomien. D'après Jonin, 1981. Tirets : failles majeures; pointillé : limite orientale du département d'Ille-et-Vilaine (et de la Bretagne).

Figure 1: The Cadomian batholith. Dashed lines: major faults; dotted line: eastern limit of Dept. Ille-et-Vilaine (and of Brittany). 
sant méridional du pluton - au profil relativement adouci par rapport aux flancs granitiques abrupts des autres secteurs - dans le bourg même et à proximité du cimetière. Le plus souvent à grain moyen mais parfois grossier, le leucogranite passe vers son extrémité orientale à un faciès à grain fin (Laouénan, 1983). Des appellations imagées (" siège et griffes du diable ", "pied de Saint-Michel ») s'efforçaient naguère d'interpréter les formes dues à l'érosion météorique, affectant le granite (Chauris, 2009).

La principale carrière était ouverte au nord du cimetière (fig. 2). En 1935, le granite livrait des pierres de taille pour la construction, des pavés, du ballast et du sable (Répertoire des carrières et industries annexes, édit. "Mines et carrières ", 1935). Ces différents produits suggèrent que toute la roche, saine ou altérée, était valorisée. La grande période d'exploitation paraît avoir été la première partie du $\mathrm{xx}^{\mathrm{e}}$ siècle. Les fronts de taille, aujourd'hui encore bien dégagés, montrent des masses de puissance métrique, recoupées par un système de diaclases très redressées. Par suite de l'altération météorique, certaines surfaces de la carrière offrent une curieuse teinte brun-rougeâtre.

Au vu des volumes excavés, la production du granite extrait au Mont-Dol a dû être considérable. La roche était déjà mise en œuvre dans l'église du bourg, construite au moins en partie aux XII ${ }^{\mathrm{e}}-\mathrm{XIV}^{\mathrm{e}}$ siècles; elle était aussi utilisée dans l'habitat, souvent marqué par la nuance brun-rougeâtre singulière des élévations. Très tôt, Dol - cité épiscopale a disposé des carrières du Mont-Dol (Leguay, 1980 et 2001) qui étaient, semble-t-il, en concurrence avec celles des îles
Chausey. Les schistes gréseux métamorphiques de contact, très sombres, ont été aussi employés sur place en moellons, dans les maisons du bourg ainsi qu'en calage dans l'église.

Divers documents réunis par J.-J. Chartier (2006), confirment également l'importance et l'ancienneté de l'exploitation de ce granite. Lors d'une réunion tenue à Dol en 1700 au sujet des digues de la baie du Mont-Saint-Michel, il est annoncé que l'« on prendra de la pierre au Mont-Dol ». Les délibérations du bureau de l'évêché de Dol (1781-1790) indiquent l'exploitation d'une carrière au Mont-Dol pour l'entretien de la banlieue de la ville. Le conseil municipal mont-dolois réuni le 20 germinal an XII (9 avril 1804) demande d'extraire " autant de pierres qu'il sera nécessaire pour l'utilité de la grande route, bâtiments publics et travaux de digues »... Ces extractions prolongées n'étaient pas sans inquiéter les amoureux du site. En 1928, le Malouin Étienne Dupont s'indignait : «Le Mont-Dol s'en va par morceaux [...]. Mutilé, amoindri, tailladé, [il] s'effrite peu à peu et, dans cent ans, un écriteau mélancolique se penchera sur le bord d'une carrière abandonnée : "Ici s'élevait le Mont-Dol." "

Effectivement, la carte dressée par l'abbé Descottes en 1922 (in Charrier, 2006) ne laisse pas d'impressionner par le nombre de carrières échancrant ses flancs. (Les deux grandes carrières de G. Faisant, d'orientation subméridienne, exploitaient le puissant filon de dolérite recoupant le granite - G. F., fig. 2). Il va sans dire qu'aujourd'hui toutes les extractions ont cessé et que la sombre prédiction d'É. Dupont ne s'est pas réalisée... Les abrupts des fronts de taille sont livrés aux adeptes de l'escalade.
Figure 2 : Localisation des carrières ouvertes dans le Mont-Dol. D'après l'abbé Descottes, 1922, simplifiée, in Charrier 2006. CF : carrière Faisant exploitant le filon doléritique subméridien; tireté : chemins.

Figure 2: Location of the quarries opened in Mont-Dol. CF: quarries Faisant exploiting the submeridian doleritic vein; dashes: ways.

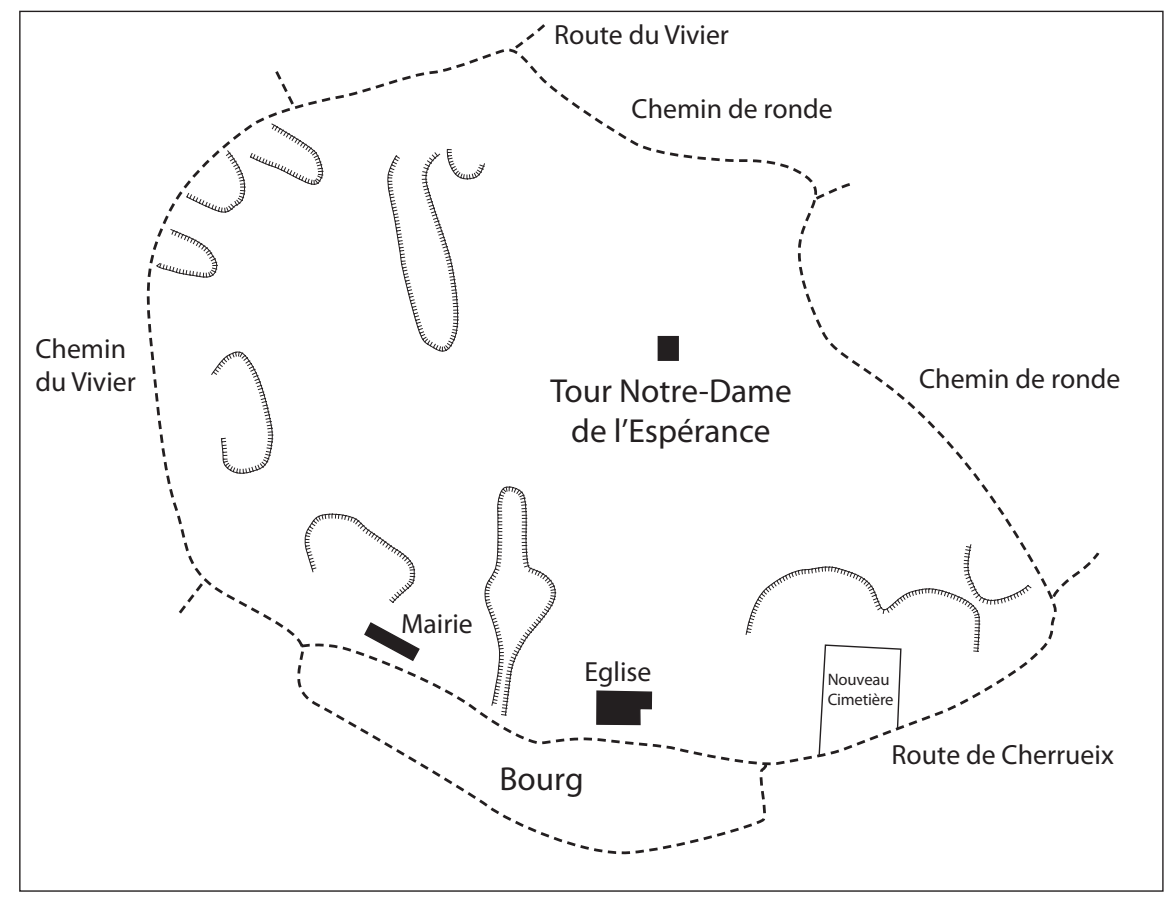




\section{Le granite de SaINT-Broladre}

Géologiquement, le pluton de Saint-Broladre présente les caractères suivants : contour ovoïde remarquable; auréole de métamorphisme de contact transformant les formations schisto-gréseuses du Briovérien en cornéennes très compactes; injection de plusieurs filons subméridiens de dolérite au début de l'époque carbonifère; nette mise en relief, particulièrement sur son flanc septentrional qui coïncide avec une ancienne falaise-morte.

Pétrographiquement, le granite offre un grain moyen à légère tendance porphyroïde, à cordiérite et biotite, avec petites enclaves; sur les bordures du massif, il s'enrichit en quartz et en muscovite. Sain, il présente une légère nuance gris-bleuté; altéré, cas le plus fréquent, il est brunâtre et d'aspect peu attrayant. La toponymie se fait parfois l'écho des affleurements : Le Rocher Ernoul, Le Rocher...

Économiquement, ce granite est susceptible de fournir des pierres de taille; plus souvent, des moellons hétérométriques. Aujourd'hui, les carrières sont abandonnées; elles étaient ouvertes à Monlieu et à La Trillardière (Roz-sur-Couesnon), à Lève-Davy (Saint-Broladre) et à La Cure (Sains; fig. 3). Par contre, sur la bordure septentrionale du massif, les cornéennes ont été exploitées et le sont toujours localement dans de grandes carrières pour l'obtention de granulats (Pensa à Baguer-Pican; La Rivière à Saint-Broladre).

Le granite de Saint-Broladre représente un exemple de ces granites bretons aujourd'hui oubliés qui pourtant, naguère, ont marqué profondément l'environnement bâti, non seulement sur leur terroir d'affleurement - ce qui, somme toute, est normal - mais aussi, pour partie, aux alentours, par suite de la rareté d'autres massifs granitiques, comme l'atteste, très tôt, son emploi dans la cathédrale de Dol. (Chauris, sous presse a).

Dans les édifices religieux, il est souvent associé à d'autres roches; ainsi dans ladite cathédrale, il côtoie les schistes briovériens proximaux, le leucogranite du Mont-Dol, le granite de Lanhélin... La dualité granite de Saint-Broladre - schistes briovériens est une des caractéristiques des édifices à l'entour du pluton (Baguer-Pican, Pontorson...) et même à une cer-

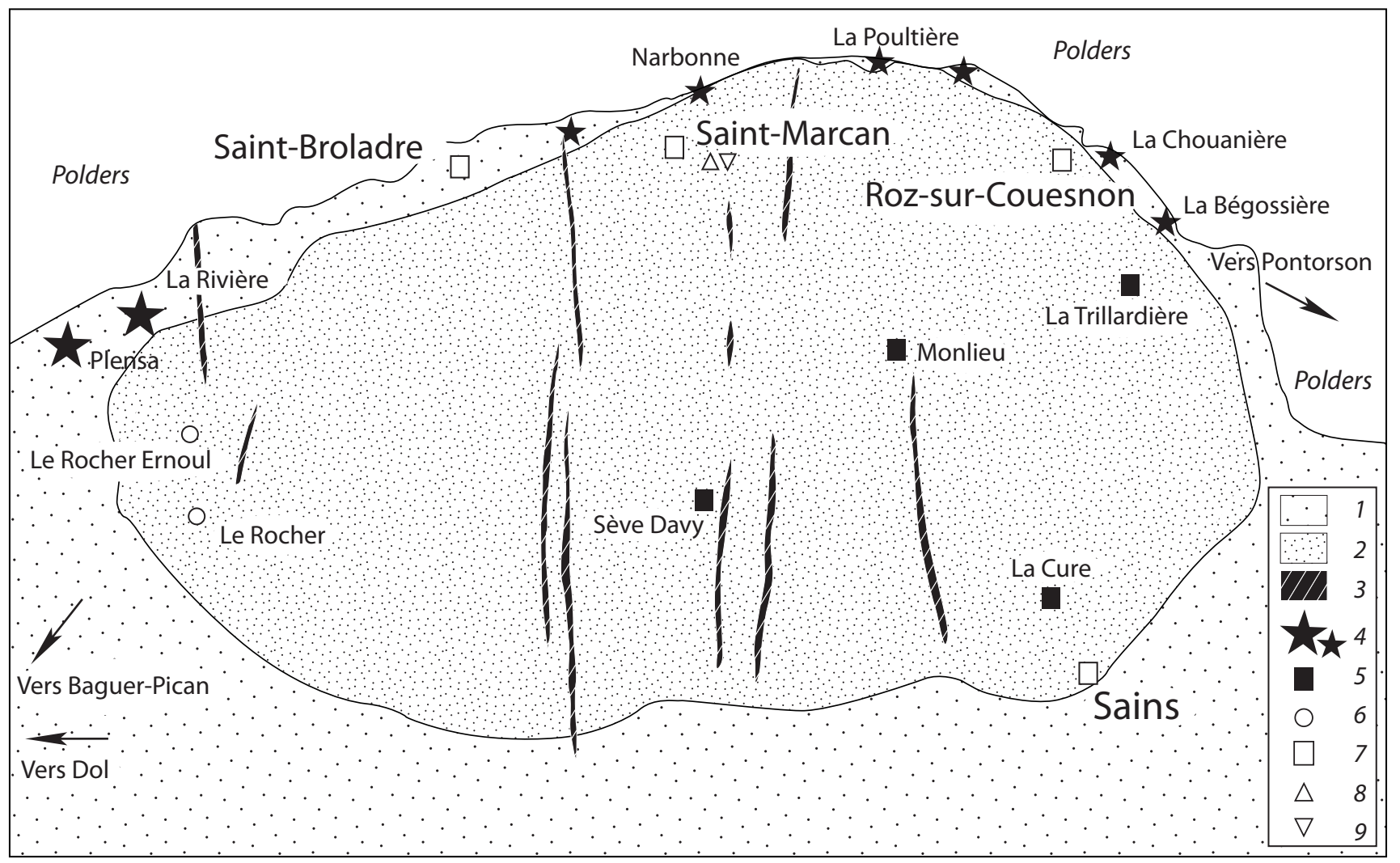

Figure 3 : Massif granitique de Saint-Broladre. D’après la carte géologique au 1/50 000 « Baie du Mont-Saint-Michel » (208), simplifiée. Localisation des lieux cités dans le texte. 1, schistes briovériens; 2, granite de Saint-Broladre; 3, filon de dolérite; 4, carrière dans les cornéennes; 5 , carrière dans le granite; 6 , toponyme significatif; 7, église paroissiale; 8, menhir; 9, croix.

Figure 3: Saint-Broladre granitic massif, simplified contours after the 1/50000 geological map, "Baie du Mont-Saint-Michel" (sheet 208), with location of place names mentioned in text. 1, Brioverian schists; 2, Saint-Broladre granite; 3, dolerite vein; 4, hornfels quarry; 5, granite quarry; 6, significant place name; 7, parish church; 8, menhir; 9, cross. 
taine distance de celui-ci (chapelle de La Coudre en SaintMéloir-des-Ondes). Un autre aspect de ces constructions est l'utilisation des faciès les plus sains (à nuance gris-blanc) pour la pierre de taille et des faciès plus ou moins altérés (brunâtres) pour les moellons hétérométriques (églises de Saint-Marcan, de Saint-Broladre). À Saint-Georges-deGréhaigne, les pierres de taille proviennent au moins en partie de Chausey; l'appel à ce granite distal s'interprète par le fait que les moines du Mont-Saint-Michel - qui avaient largement utilisé ce granite insulaire pour leur abbaye avaient érigé ici un prieuré.

Des remarques comparables peuvent être effectuées pour l'habitat. À Saint-Marcan, les maisons du bourg, par leur teinte brunâtre due à l'emploi généralisé de la pierre locale, présentent un aspect assez triste; localement, le granite, fissuré, sert même de soubassement au bâti, qui est ainsi une émanation directe du sous-sol. De même, à Roz-sur-Couesnon, les vieilles maisons en granite du bourg montrent un habitus brunâtre, reflet des pierres de taille d'encadrement des ouvertures et des moellons des élévations. À Baguer-Pican, un mur de maison expose la triple association schiste briovérien, granite de Saint-Broladre et dolérite. Le granite de Saint-Broladre a été recherché au moins jusqu'à Cancale comme l'atteste son emploi dans la porte cintrée d'une vieille demeure à La Ville-Jégu. Le menhir de Saint-Marcan a évidemment fait appel à la roche locale. La vieille croix, dite "Croix-Seigneur ", fournit un exemple exceptionnel des aptitudes du granite de Saint-Broladre; l'édicule, en deux éléments - dont la partie supérieure ou croix sensu stricto présente des cupules - a mis en œuvre le faciès brunâtre un peu porphyroïde.

\section{Le GRANITE DE DingÉ}

La célébrité du granite de Dingé est ancienne. Vers le milieu du Xvi ${ }^{e}$ siècle, Noël du Fail, décrivant la maison d'un paysan, précise que « la paroi ne fut jaspe, porphyre, marbre, encore moins [...] tuffeau, querignan ou dingué... ". Son aptitude à fournir de belles pierres de taille, que livraient de nombreuses carrières, se traduit par l'élégance des constructions édifiées sur le massif granitique et aux alentours mais, depuis longtemps, les extractions ont cessé, les carrières sont noyées ou envahies par la végétation arborescente, voire comblées. Les dimensions du massif sont relativement modestes : environ 9 kilomètres d'ouest en est sur un peu plus de 3 kilomètres au maximum du nord au sud. Il apparaît légèrement en relief au-dessus des schistes briovériens métamorphisés en cornéennes à son contact, en particulier à son extrémité occidentale où le dénivelé est de l'ordre d'une quarantaine de mètres. En divers points, la toponymie, tout en soulignant la topographie (Le Tertre, Le Tertre Odie...) indique la présence d'affleurements rocheux (Rochetain, La Motte-au-Rocher, La Roche-Bernard, Le Rocher-Aoustin, Le Rocher-Taupin, La Pierre-Plate, Les Hauts-Rochers...) avec çà et là, présence de boules (PlessisBriand, La Chiffardière...; fig. 4).

La plus grande partie du pluton est constituée par un granite avec enclaves assez fréquentes, à grain moyen voire légèrement porphyroïde, à cordiérite et à biotite. Il offre une teinte bleutée qui n'est pas sans rappeler celle du granite de Lanhélin affleurant largement plus au nord, si bien que dans certaines constructions une confusion reste possible. Vers son extrémité orientale, tant au nord (Vauluisant) qu’à l'est (La Bouderie), ce faciès fait place à une variété à granulométrie plus fine, enrichie en muscovite avec un peu de tourmaline, appartenant à la famille des leucogranites. De nombreux filons de dolérite recoupent le granite de Dingé et le Briovérien encaissant. Cette roche, de nuance vert-noirâtre, très tenace et offrant une altération en boules, était naguère très estimée - sous le nom de " pierre de fer » - pour l'empierrement des chemins.

La répartition des carrières de granite ouvertes dans le massif de Dingé s'avère à première vue assez curieuse : si elles sont presque toutes localisées vers les bordures du pluton, elles sont toutefois essentiellement concentrées vers son extrémité occidentale (au moins neuf carrières dont celles de La Ville-Geffrend) et son extrémité orientale : La Bouderie (cinq), Les Hauts-Rochers (deux), Vauluisant (deux); elles sont rares vers la bordure septentrionale (Le Vaupicot, une; Le Rouvre, une) et, à notre connaissance, absentes dans sa bordure méridionale. Une telle répartition peut s'expliquer, au moins en partie, par la topographie : la bordure du pluton se traduit, comme déjà noté, en particulier à l'ouest, par une rupture de pente qui, en favorisant l'érosion, tend à débarrasser la roche de son manteau d'altérites, facilitant ainsi l'extraction. Cette hypothèse trouve confirmation dans l'absence de carrières le long de la bordure méridionale où, le plus souvent, le dénivelé est très faible. Toutefois, l'impact topographique n'est certainement pas seul à intervenir : l'abondance des carrières dans la partie orientale du massif est à rapporter à la présence du faciès à muscovite, plus facile à façonner que le faciès à biotite seule. Par ailleurs, facteur économique, on peut aussi se demander si la fréquence des exploitations à la bordure ouest n'est pas due à la proximité du canal d'Ille-et-Rance qui, aux époques où les charrois par de mauvais chemins restaient pénibles et dispendieux, offrait un moyen de transport économique et aisé; ce point mériterait d'être éclairci. La morphologie des carrières est différente selon les points; certaines sont très allongées (carrière nord de La Ville-Geffrend; carrière de La Bouderie). Les dates d'arrêt des extractions ne nous sont pas connues 


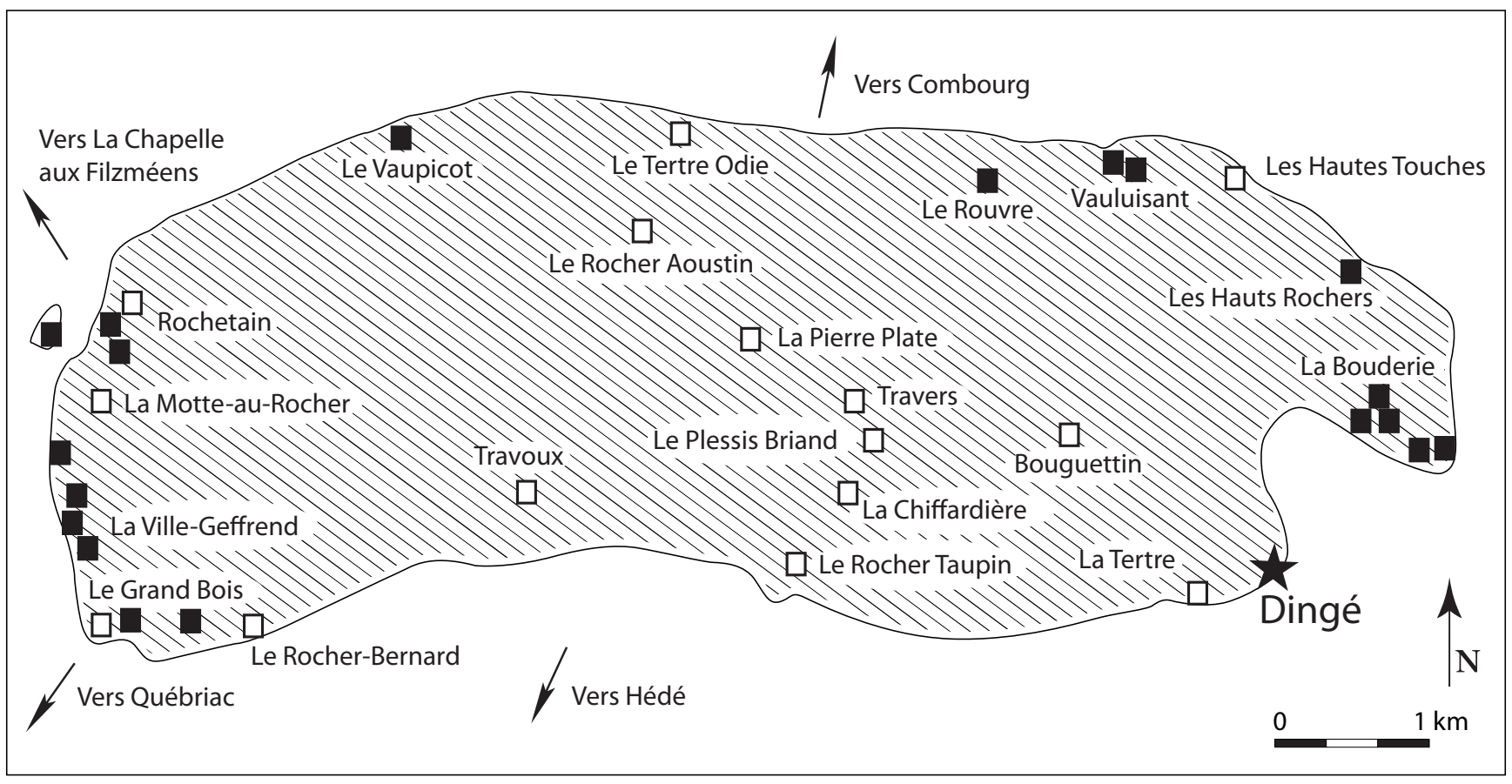

Figure 4 : Massif granitique de Dingé. Contours d'après les cartes géologiques au 1/50 000 "Caulnes » (281) et « Combourg » (282), simplifiées. Carrière : carré noir. Lieux-dits cités dans le texte : carré blanc. Les filons de dolérite n’ont pas été figurés.

Figure 4: Dingé granitic massif; simplified contours after the 1/50000 geological map, "Caulnes" (281) and "Combourg" (282) sheets. Quarry: black square; place name mentioned in text: clear square. Dolerite veins not reported.

avec précision. Selon nos informateurs oraux, la carrière de La Ville-Geffrend aurait cessé son activité lors de la Grande Guerre; à La Bouderie, l'exploitation se serait poursuivie jusque vers les années 1960. (Chauris, sous presse b).

Comme on pouvait s'y attendre, l'église de Dingé (seconde partie du XIX siècle) a fait appel, tant pour la pierre de taille (localement desquamée) que pour les moellons, au granite à muscovite local. Par contre, tout à fait inattendue est la mise en œuvre, pour le portail, d'un calcaire jaunâtre dont la provenance n'est pas assurée (pierre de Caen?). Pour l'église de Québriac, édifiée à un peu plus d'un kilomètre seulement du massif de Dingé, le granite bleuté proximal, à grain moyen, légèrement porphyroïde, a été tout naturellement recherché; les moulures du portail attestent ses qualités. Toutefois, la pyrite entraînant l'apparition de taches de rouille et les enclaves sombres atténuent la beauté de certaines pierres... À La Chapelle-aux-Filzméens, très proche aussi du pluton, appel a également été fait au granite local, bleuté et à tendance porphyroïde; les moellons revêtent par altération une coloration brunâtre peu engageante... La diversité lithologique de l'église de Saint-Domineuc est liée aux différentes époques de construction, du XIII ${ }^{\mathrm{e}}$ siècle au XIX ${ }^{\mathrm{e}}$, avec remploi de moellons en schistes briovériens, dolérite, calcaire...; le soubassement de la tour est probablement en granite de Dingé, affleurant à 4 kilomètres seulement plus à l'est.
La monumentale église de Tinténiac, de style néo-byzantin, édifiée au tout début du $\mathrm{Xx}^{\mathrm{e}}$ siècle sur les plans d'Arthur Regnault, (Andrieux, 2011), nécessite de plus longs développements par suite de la diversité des pierres ici mises en œuvre. Selon le devis descriptif du 29 octobre 1898, le granite sera pris dans les carrières de Hédé et de Saint-Brieuxdes-Iffs (massif de Bécherel) ainsi que de Québriac (massif de Dingé) : "On choisira de préférence celui qui offrira le plus de lit et de queue. " Dans un devis en date du 31 mai 1901, en sus du granite, il est question des calcaires de Caen (Normandie) et de Sireuil (Charente)... Dans la récapitulation des travaux et dépenses établies par A. Regnault, il est fait mention de granite ainsi que de ces deux calcaires ${ }^{2}$. Nos observations in situ confirment la participation importante du granite de Dingé (carrières de Québriac), nettement bleuté, avec dissémination de feldspaths légèrement porphyroïdes et nombreuses petites enclaves sombres. Un portail latéral en clair granite à grain fin de Languédias est, selon toute probabilité, un remploi de l'ancien édifice.

L'aptitude au façonnement du granite fin à muscovite extrait dans la partie orientale du pluton de Dingé explique

2. Qu'il nous soit permis de redire ici nos vifs remerciements à Dominique Provost (Patrimoine Tinténiac-Montmuran) pour les informations transmises à l'issue de ses recherches dans les archives du presbytère de Tinténiac et de ses enquêtes orales. 
sa large utilisation pour l'habitat assez loin vers l'ouest. Dans le bourg de Dingé, une maison à portes géminées, où le cintre externe joue le rôle d'arc de décharge, met en œuvre de manière aléatoire le faciès bleu très sain et le faciès beige légèrement altéré. Constatations comparables dans plusieurs autres maisons du bourg; il n'apparaît pas de différences significatives dans la recherche de l'une ou l'autre variété de coloration pour les pierres de taille et les moellons. Les mêmes observations peuvent être effectuées à La Bouderie, aux Hauts-Rochers, à Vauluisant, La Chiffardière, Le Rocher-Taupin, La Pierre-Taillée... Plus à l'ouest, le granite à grain moyen est largement employé (La Morandière, Le Tertre-Odie, Travoux, Le Grand-Bois...), parfois associé au granite à grain fin.

En dépit de quelques incertitudes, dues entre autres à une certaine similitude de faciès avec le granite de Lanhélin, les annotations présentées ici attestent l'impact du granite de Dingé dans les constructions, ce que laissaient d'ailleurs présumer les nombreuses carrières depuis longtemps abandonnées.

\section{Le GRANITE de BÉCHEREL}

Les traits majeurs caractérisant le granite de Bécherel peuvent être succinctement présentés de la manière suivante (Chauris, 2010).

- Structuralement, un allongement est-ouest de l'affleurement, démesuré (près de $30 \mathrm{~km}$ ) par rapport à la largeur (souvent 2 à $3 \mathrm{~km}$ seulement), soit dans un rapport de l'ordre de 10 (fig. 5) ; une intrusion dans les schistes briovériens, transformés par métamorphisme de contact en cornéennes et schistes tachetés; un contact par failles, en plusieurs points au sud, avec les terrains paléozoïques; des modifications par le passage de l'accident linéamentaire (ou faille majeure) médio-armoricain qui se traduit, entre autres par la formation de puissants filons quartzeux, orientés est-ouest, en sus de la cataclase ou bréchification du granite; enfin, présence de nombreux filons de dolérite. Une grande carrière abandonnée, envahie par la végétation et rendue dangereuse par suite d'éboulements, exploitait le granite de Bécherel à proximité de Hédé, près du carrefour du Perray. Une petite carrière était ouverte dans la dolérite en bordure du chemin allant de Bécherel à Miniac près du lieu-dit Lessart; le filon doléritique qui recoupe le granite de Bécherel atteint ici une vingtaine de mètres de puissance et présente la classique altération en boules. L'immense carrière de La Rodais, située à l'est de Guenroc, est ouverte dans le Briovérien métamorphique et le granite écrasé de Bécherel.

- Pétrographiquement, une texture à grain moyen (quelques millimètres), avec biotite, cordiérite altérée (en pinite) et un peu de pyrite disséminée; fréquence de petites enclaves schisteuses du Briovérien métamorphique, ainsi que d'enclaves quartzeuses. Quand la roche est saine, ce qui est rare, elle offre une légère nuance bleutée; en général, l'altération météorique se traduit par une teinte brunâtre due à la diffusion d'hydroxyde de fer du type limonite, liée à la décomposition des minéraux ferrifères (biotite, pyrite). Cette décomposition in situ, pouvant entraîner une intense arénisation parfois sur une dizaine de mètres de profondeur, n'a pu que s'accentuer dans les constructions.

- Géomorphologiquement, un net relief est souligné par une rupture de pente à Hédé ainsi que par les hauteurs de Bécherel, dominant de plus d'une centaine de mètres la dépression creusée au nord dans les schistes briovériens.

- Économiquement, une exploitation fut effectuée naguère dans de nombreuses carrières, ouvertes tant dans le granite lui-même (pour moellons et pour arènes) que dans les filons quartzeux qui le recoupent et dans les cornéennes de contact qui le bornent (principalement pour l'empierrement).

Les aptitudes architectoniques médiocres du granite de Bécherel (teinte brunâtre peu agréable, cataclase, fréquence des enclaves...) ont conduit souvent les bâtisseurs d'églises à faire appel, en sus, à d'autres roches, plus ou moins lointaines mais de meilleure qualité (fig. 6). C'est ainsi qu'ont été recherchés les granites du massif de Dingé (à nuance bleutée) et ceux du massif hercynien de Dinan (Le Hinglé, à grain moyen, très légèrement bleuâtre; Brusvily, aux longs feldspaths blanchâtres; Languédias, à grain fin, blanc-gris clair). Le clair calcaire miocène dit "pierre de jauge ", extrait aux environs du Quiou (Chauris, 2006), a été également utilisé. Plus surprenant au premier abord, la mise en œuvre du tuffeau du Val-de-Loire dans quelques édifices récents s'explique par la facilité de son façonnement et la proximité du canal d'Ille-et-Rance. Enfin, pour les moellons des édifices érigés au-delà des limites du granite de Bécherel, appel a pu être fait également soit aux sombres schistes briovériens, soit aux grès paléozoïques blanchâtres... Les annotations qui suivent se limitent à quelques édifices religieux (fig. 5).

En dépit de remaniements aux $\mathrm{XVI}^{\mathrm{e}}$, XVII ${ }^{\mathrm{e}}$ et $\mathrm{XIX}^{\mathrm{e}}$ siècles, Notre-Dame à Hédé reste encore aujourd'hui la plus grande église romane d'Ille-et-Vilaine. Sa construction à une époque reculée explique à la fois l'utilisation du granite local, très largement mis en œuvre, et son degré d'altération avancée. Dans l'élévation méridionale, sous le crépi, les moellons, en granite brunâtre très érodé, sont réunis par un mortier terreux jaunâtre. Dans la façade occidentale, le granite, en moellons assisés, brunâtre, avec enclaves de quartz et de roches métamorphiques, offre une nette érosion en creux par rapport aux joints. Les éléments moins altérés laissent entrevoir une légère nuance bleu-gris. La pierre des contre- 


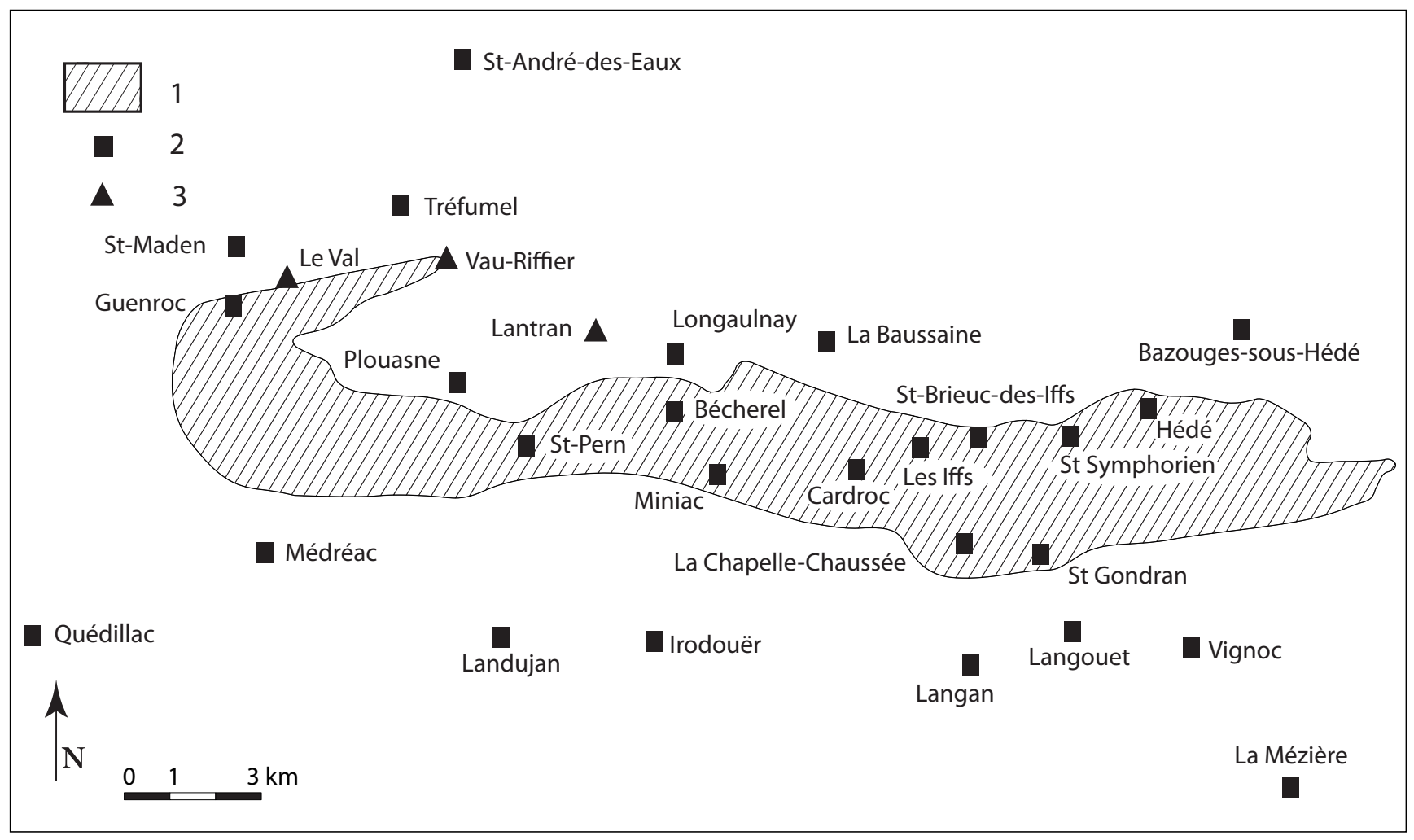

Figure 5 : Édifices religieux ayant fait appel au moins pour partie au granite de Bécherel (1). 2 : église. 3 : chapelle (enquêtes inachevées). Figure 5: Religious buildings utilizing (at least pro parte) the Bécherel granite (1); 2, church; 3, chapel (enquiries still in course).

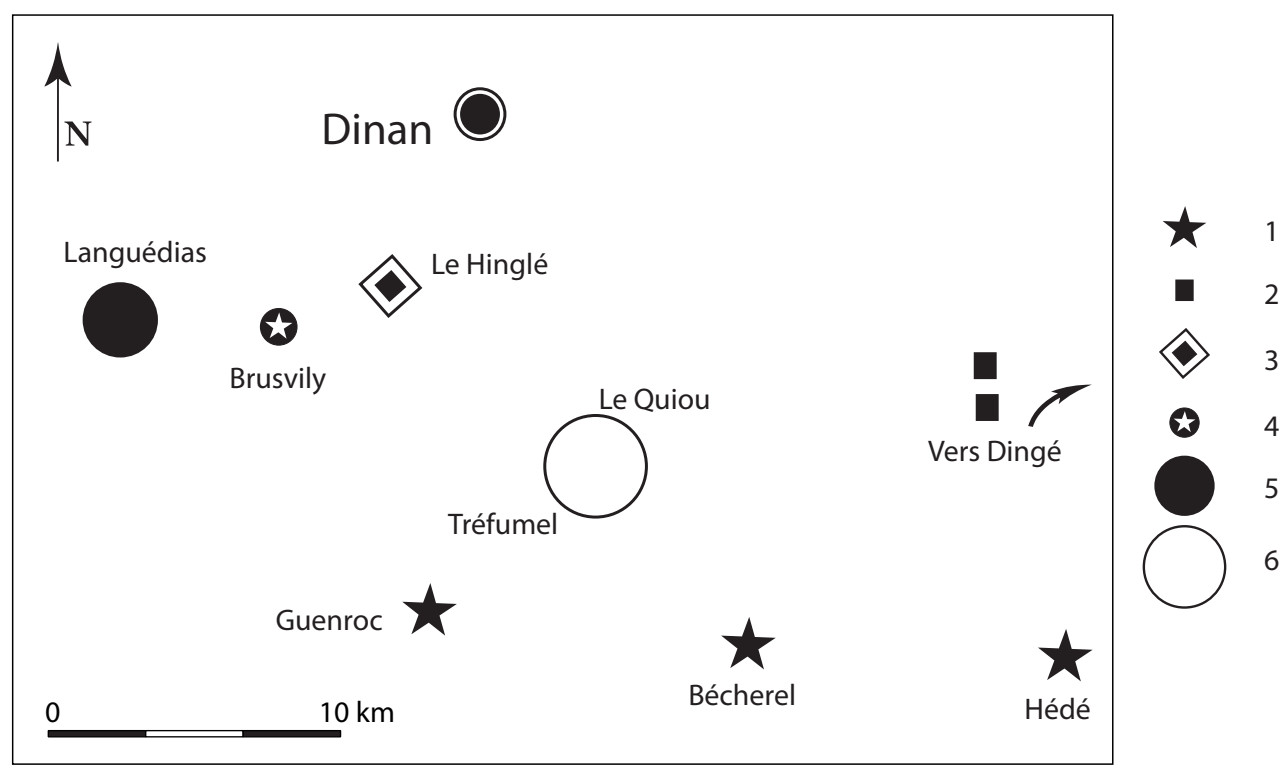

Figure 6 : Provenance (schématisée) des principales roches mises en ouvre dans les édifices religieux ayant fait par ailleurs appel au granite de Bécherel (1); granite de Dingé (2) ; granites hercyniens du Hinglé (3), de Brusvily (4), de Languédias (5); calcaire du Quiou (6). Les autres types de matériaux n'ont pas été indiqués.

Figure 6: Schematized provenance of the main rocks used in the religious buildings built with Bécherel granite (1); Dingé granite (2); hercynian granites of Le Hinglé (3), Brusvily (4), Languédias (5); Le Quiou limestone (6). Other types of materials not reported. forts - plus tardifs et remontant seulement au XVII ${ }^{\mathrm{e}}$ siècle est mieux façonnée.

Dans l'église Notre-Dame à Bécherel, de l'édifice du $\mathrm{XVII}^{\mathrm{e}}$ siècle, seul subsiste à présent le porche; la reconstruc- tion, sur les plans de l'architecte Jacques Mellet, a eu lieu en 1868-1870; le clocher, dû à Arthur Regnault (Andrieux, 2011) remonte seulement à 1898. Comme on pouvait s'y attendre, large appel a été fait au granite local, soit en 
pierres de taille (façade ouest), soit en moellons. À ce matériau proximal s'associent localement le calcaire du Quiou et, parfois, un peu de granite de Languédias, tous deux probablement en remploi de l'ancien bâti. L'intérieur de l'église ne laisse pas de surprendre avec la mise en ouvre pour les piliers du clair tuffeau du Val-de-Loire. Au-dessus du porche, la niche centrale est occupée, depuis 2001, par une statue de la Vierge, en marbre de Carrare (Italie).

Après le $\mathrm{XII}^{\mathrm{e}}$ siècle, de multiples transformations, aux $\mathrm{Xv}^{\mathrm{e}}$ et XVII ${ }^{e}$ siècles, ont modifié l'église de Miniac-sous-Bécherel. Les élévations sont en granite brunâtre de Bécherel, utilisé également dans la tourelle du clocher dont le cordon est toutefois en granite de Languédias. L'élégante porte occidentale surbaissée est aussi en granite de Languédias, de même que la porte méridionale (1625)... L'emploi d'un bloc de quartz dans la fondation sud reflète la présence d'un filon est-ouest tout proche. Dans le porche sud, notons la présence d'un banc en calcaire. Le principal intérêt lithologique de l'église est de révéler l'appel précoce au granite de Languédias pour les éléments ouvragés (Chauris, 2005).

L'église des Trois-Marie à Cardroc (XvII $-\mathrm{XIX}^{\mathrm{e}}$ siècles), en plein massif granitique de Bécherel mis en œuvre dans les murs, a fait en outre appel, non seulement au calcaire du Quiou (modillons), mais également au granite de Languédias. La dualité granite brunâtre de Bécherel - clair calcaire du Quiou se retrouve à Saint-Brieuc-desIffs (reconstruction au XVII ${ }^{\mathrm{e}}$ siècle, avec conservation de quelques vestiges romans et érection tardive d'un clocher par Arthur Regnault), ainsi qu'à Saint-Symphorien ( $\mathrm{xv}^{\mathrm{e}}$ $\mathrm{XVI}^{\mathrm{e}}$-XIX ${ }^{\mathrm{e}}$ siècles), avec emploi d'un énorme bloc de quartz dans le soubassement d'un contrefort par ailleurs en granite de Bécherel. À Saint-Gondran, aux moellons granitiques hétérométriques de Bécherel sont associés quelques éléments de grès paléozoïques qui affleurent à faible distance plus au sud. Beaucoup plus intéressante y est la présence sporadique d'un microgranite de teinte beige avec quelques phénocristaux feldspathiques, alors que les cartes géologiques, tant au $1 / 80000$ qu'au $1 / 50000^{\circ}$, n'indiquent aucun filon de ce type aux environs : une fois de plus, l'examen attentif du bâti incite les géologues à affiner leurs observations sur le terrain. Même constatation à La Chapelle-Chaussée où un microgranite a été observé parmi les moellons en granite de Bécherel.

Avec l'éloignement du massif de Bécherel, la part de cette pierre tend à décroître. Tel est le cas de l'église de Vignoc : si ledit granite est encore ici utilisé, l'édifice montre un large emploi des grès blancs paléozoïques qui affleurent à proximité; ces grès livrent non seulement des moellons mais aussi des pierres de taille (pour partie dans une porte sud où le grès présente de jolis cernes). Dans l'église de La Mézière (reconstruction en 1868-1871, excepté la tour de 1822-
1833), plus éloignée du granite de Bécherel, une partie des moellons des élévations provient encore de ce massif, en association avec des grès paléozoïques. Selon toute probabilité, c'est au granite bleuté de Dingé, dont l'acheminement était facilité par le canal d'Ille-et-Rance, qu'il faut rapporter les pierres de taille (contreforts...). Le grand portail est en tuffeau du Val-de-Loire.

Les observations présentées sur des édifices religieux ayant mis en œuvre le granite de Bécherel en association avec d'autres roches peuvent l'être également sur l'habitat. À Hédé comme à Bécherel, les demeures sont caractérisées par l'emploi du granite local qui leur confère une tonalité brunâtre un peu triste. Le polylithisme, se retrouve également dans l'habitat. À Plouasne, l'ancien presbytère restauré associe granite de Bécherel, calcaire du Quiou et granite de Languédias. À Miniac-sous-Bécherel, dans une vieille demeure du bourg, les portes géminées, à double cintre, en clair calcaire caverneux riche en fossiles, tranchent vivement sur les sombres moellons granitiques de Bécherel; fait à signaler, contrairement aux cas les plus fréquents, les portes sont ici en calcaire et non en granite de Languédias. À Guenroc, une maison ancienne présente un soubassement formé de gros blocs de quartz blanchâtre, surmontés par des moellons hétérométriques en granite de Bécherel. Dans les dépendances du manoir de Champsavoy, au sud-sud-est de Saint-Judoce, se note l'association du granite de Bécherel aux schistes briovériens, au calcaire du Quiou et au granite de Languédias...

À l'inverse de nombreux granites bretons, la pierre de Bécherel ne convient guère au grand appareil. Ses nombreuses enclaves de schiste, voire de quartz, et surtout la cataclase qui l'affecte, la rendent, en règle générale, inapte au façonnement élaboré; par ailleurs, sa teinte brunâtre, due à une profonde altération, n'est effectivement pas très attractive... Son principal atout reposait sur son abondance, inépuisable. À défaut de qualité, la quantité! Par ailleurs, son emploi fréquent au-delà des limites du massif, tant au nord qu'au sud, est en grande partie la conséquence d'une absence de granites dans ces secteurs où dominent des roches schisteuses, le plus souvent fort médiocres elles aussi. Dans ces conditions, l'appel à d'autres roches régionales s'interprète aisément. Cette association introduit dans les constructions un polylithisme qui, en un certain sens, tempère - mieux atténue - la médiocrité du granite dominant. Tel est le cas du granite de Languédias et, peut-être plus encore, du calcaire du Quiou. Finalement, le granite de Bécherel s'oppose totalement au granite, pourtant voisin, de Dingé qui, lui, a fourni de superbes pierres de taille offrant une agréable nuance bleutée. 


\section{LE GRANITE DE LOUVIGNÉ-DU-DÉSERT}

Seule la partie sud-ouest de l'immense pluton dit de Louvigné-Gorron est située dans le département d'Ille-etVilaine; les autres, localisées dans les départements de la Manche et de la Mayenne, restent, par suite, hors de nos propos limités à la Bretagne. On notera d'ailleurs que la plupart des carrières sont ouvertes dans la zone «bretonne " de ce pluton, à savoir dans les bassins dits de Louvigné sensu stricto (au nord) et du Coglais (au sud-ouest; fig. 7).

Le granite de Louvigné-du-Désert, isogranulaire, à grain moyen, offre une nuance gris-bleu. Le quartz gris et les lamelles noires de biotite s'y détachent sur un fond feldspathique clair (feldspaths potassique et calco-sodique à cœur d'andésine et à bordure d'oligoclase); la densité est de 2,70. Aux affleurements et dans certaines carrières, la roche pré- sente localement la classique altération en boules plus ou moins dégagées par l'érosion du manteau arénitique. $\mathrm{Au}$ Bois de La Vaine, à Mellé, la Roche-du-diable est un bel exemple d'empilement de boules; à Monthault, d'autres boules se notent aux Bruyères-du-Bois-Veil; de même aux Couardes à Saint-Germain-en-Coglès; à La Potelais en Coglès, la "grotte de Lourdes " a été aménagée dans une accumulation de boules. Les néolithiques ont mis à profit ces formes d'érosion, comme l'atteste, entre autres, l'allée couverte du Rocher-Jacquet au sud-est de Saint-Germainen-Coglès (Cucarull, 2001).

Voici encore une cinquantaine d'années, le granite était ici l'objet d'une exploitation intense (Pierre, 1962). Vers les années 1960, 115 établissements granitiers étaient en activité dans le bassin de Louvigné. Sur ce nombre, environ 80 étaient exploités par des artisans (1 à 5 salariés); une seule

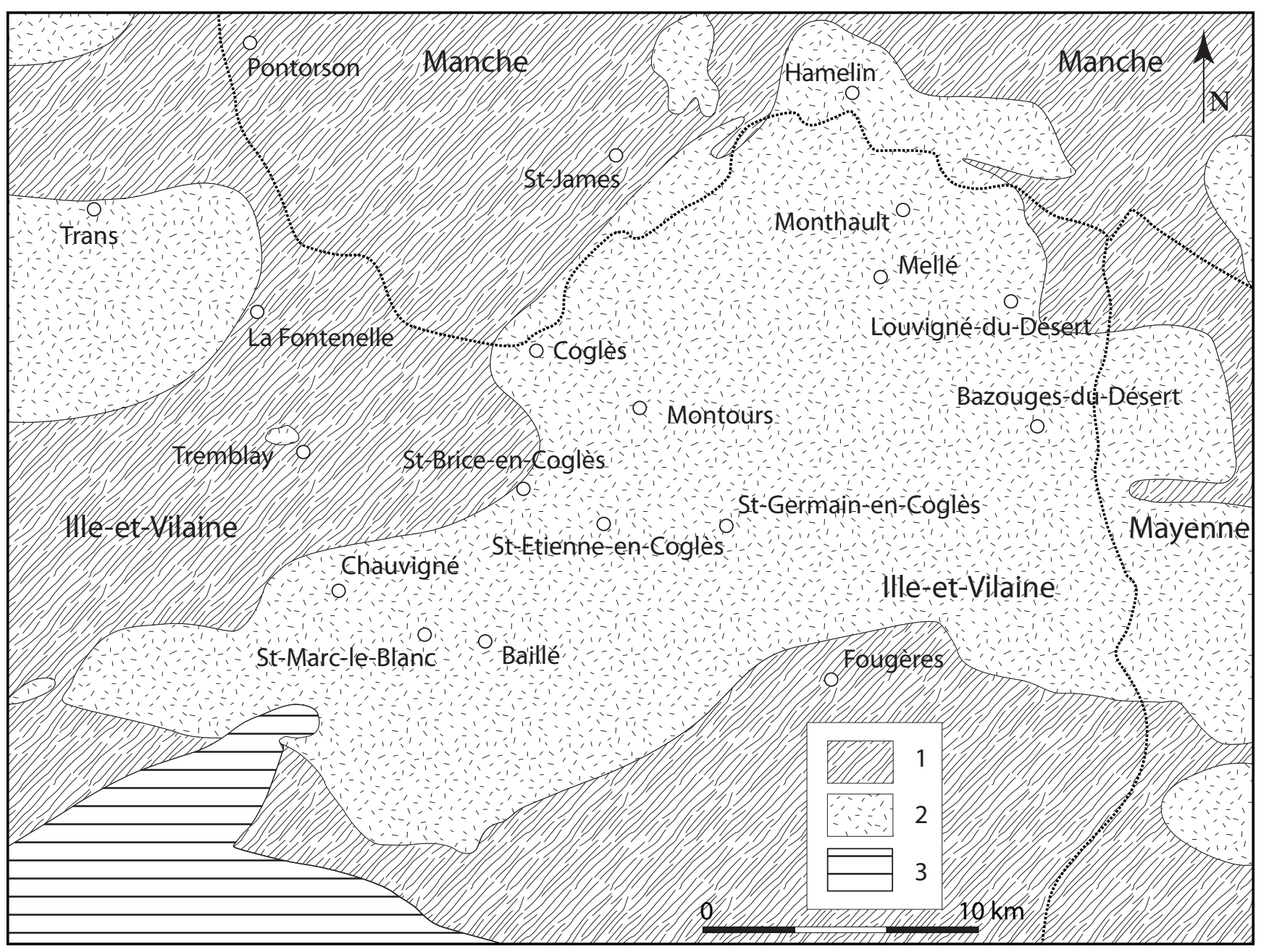

Figure 7 : Partie occidentale du pluton de Louvigné-du-Désert au nord de Fougères. Seule une partie du massif est en Ille-et-Vilaine (les tiretés marquent la limite départementale). 1 : Briovérien; 2 : Granite; 3 : Paléozoïque.

Figure 7: Western part of Louvigné-du-Désert pluton, north of Fougères (only a part of the massif is in Ille-et-Vilaine; dashed line marks the departmental limit). 1, Brioverian; 2, granite; 3, Palaeozoic. 
entreprise comptait plus de 100 salariés, deux de 50 à 100 . Dans le bassin du Coglais également, 45 des 70 entreprises granitières étaient des établissements artisanaux (5 salariés au plus). Le grand nombre des petites entreprises était dû à la nature même de la production, essentiellement des matériaux de voirie (pavés et bordures) n'exigeant pas d'énormes investissements. À présent, la poursuite de cette activité dans quelques grandes exploitations qui se sont développées sur le site même des petites carrières artisanales ne peut masquer l'arrêt d'entreprises importantes, telle "L'Avenir " à Louvigné (Joret, 2012).

Il s'avère aujourd'hui difficile d'établir la liste précise des carrières tant leur nombre était grand. Parmi les plus célèbres, évoquons toutefois, dans le bassin de Louvigné, Montlouvier, Dolent, La Beurrière, La Piochais, La Fevetière, Pierrelée, Le Val, La Morinais, La Fosse à Godard... ; dans le bassin de Coglès, les Échelles et le Haut-Rocher à SaintGermain-en-Coglès, La Roche-Hue et Le Bois-des-Rochers à Saint-Marc-le-Blanc... ; dans cette dernière exploitation, arrêtée en 1986, un dessin a été gravé en 1997 sur une des parois. À présent, les granites locaux ne fournissent plus qu'un pourcentage relativement restreint, principalement pour la voirie, des roches travaillées; une technicité de haut niveau sur des granites importés d'autres sites français ou étrangers a remplacé l'artisanat; il suffit de citer ici "La Générale du Granit », à Louvigné, les établissements Rébillon dans le Coglais.

Si le dénombrement des anciennes carrières s'avère être une tâche presque insurmontable, que dire alors de l'interminable "litanie " des ouvrages ayant fait appel à ces granites! Dans le passé, citons, parmi bien d'autres, l'église de Pontmain (Mayenne) et surtout les superbes monolithes de l'église Saint-Pierre-de-Montrouge à Paris. Après la Grande Guerre, vient le monument canadien de SaintJulien près d'Ypres en Belgique (1923) ou, encore au début du $\mathrm{Xx}^{e}$ siècle, le monument à la mémoire du syndicaliste Fernand Pelloutier au cimetière de Sèvres (Hautsde-Seine), en provenance de la carrière de Dolent (1924); le monument des Pilgrim Fathers aux États-Unis (1929) - 7,50 mètres de haut, environ 100 tonnes - provient de la carrière de Pierrelée. Après la dernière guerre, le monument Patton à Avranches, stèle pyramidale à cinq pans, haute de 14 mètres, est en granite bourchardé en provenance de Louvigné. "Le Signal ", monument élevé à Douai à la mémoire des professeurs et des élèves du lycée morts pour la France a été façonné (vers 1959) dans le granite extrait de la carrière du Val. Lors du symposium «Sculptures sur granit » tenu à Lanhélin durant l'été 1993, le sculpteur américain Prentice a dressé un monolithe de 4,50 mètres $\mathrm{x} 1,30$ mètre x 0,25 mètre en granite de Louvigné, percé sur chaque face de 31 lignes alternativement de 7 et 8 trous et intitulé « Mur du Silence ", original mégalithe contemporain. En 2009, à Saint-Pol-de-Léon, deux statues - aujourd'hui dressées dans la Vallée des Saints à Carnoët (Côtes-d'Armor) - ont été sculptées dans ce granite, l'une représentant saint PolAurélien, par David Puech, l'autre saint Patern, par Olivier Lévêque.

Aujourd'hui, le granite de Louvigné est utilisé un peu partout en France pour des travaux de voirie : voies piétonnes à Marseille, Mulhouse, Metz, Paris... ; dallages pour le tramway de Saint-Denis à Bobigny, la ZAC du Parc de Paris-Bercy, le métro à Anthony... (collectif, 1998). Le site d'origine du granite n'a pas été oublié : à Louvigné, la pierre locale a été récemment mise en œuvre pour l'aménagement de la place, tant en revêtement de sol que dans les bassins de la fontaine (anonyme, 2003).

Toutefois, l'ouvrage le plus spectaculaire jamais réalisé en granite de Louvigné est sans conteste l'ensemble des 72 colonnes à section carrée $(0,90 \mathrm{~m})$ et 4,5 mètres de haut, dressées à Rennes sur le site de Beauregard et inaugurées le 17 juin 2006. Cet étonnant ensemble mégalithique surgi à l'aube du XxI ${ }^{\mathrm{e}}$ siècle est dû à Aurélie Nemours qui définissait ainsi son travail : "Une ouvre de la terre vers le ciel, qui part de la matière pour atteindre l'esprit, comme un dialogue entre l'art et l'éternité. " Les blocs ont été extraits dans la carrière Godard à Louvigné puis fendus en éléments de $5 \mathrm{mx} 1 \mathrm{~m} \times 1 \mathrm{~m}$, avant d'être sciés sur six faces à $\mathrm{La}$ Générale du Granit (Cochard, 2006). Le mégalithisme a ressurgi en Bretagne...

\section{LE GRANITE DE LANHÉLIN}

Le pluton de Lanhélin est un massif composite présentant plusieurs faciès (fig. 8). Le plus célèbre - et de loin - correspond au granite de Lanhélin sensu stricto, isogranulaire, à grain moyen, caractérisé par sa teinte bleue accusée; le feldspath potassique (microcline) y est associé au plagioclase (limite andésine-oligoclase), à la biotite et au quartz; les enclaves sont peu nombreuses. Les fractures subméridiennes peuvent être soulignées par l'apparition d'une nuance verdâtre due à la "saussuritisation " des plagioclases et à des amas de chlorite. Quelques fractures ont été empruntées par de puissants filons de dolérite (carrière Guillou-Granits). Un granite de teinte claire affleure à la bordure de la partie orientale du pluton, entre autres à La Fontenelle. Deux petits pointements leucogranitiques recoupent le massif au sud de Trans; au Rocher-Toc, la roche admet des nodules de tourmaline; près du Tremblay, un pointement leucogranitique plus important affleure au sud-est du massif principal (Jonin, 1969). 

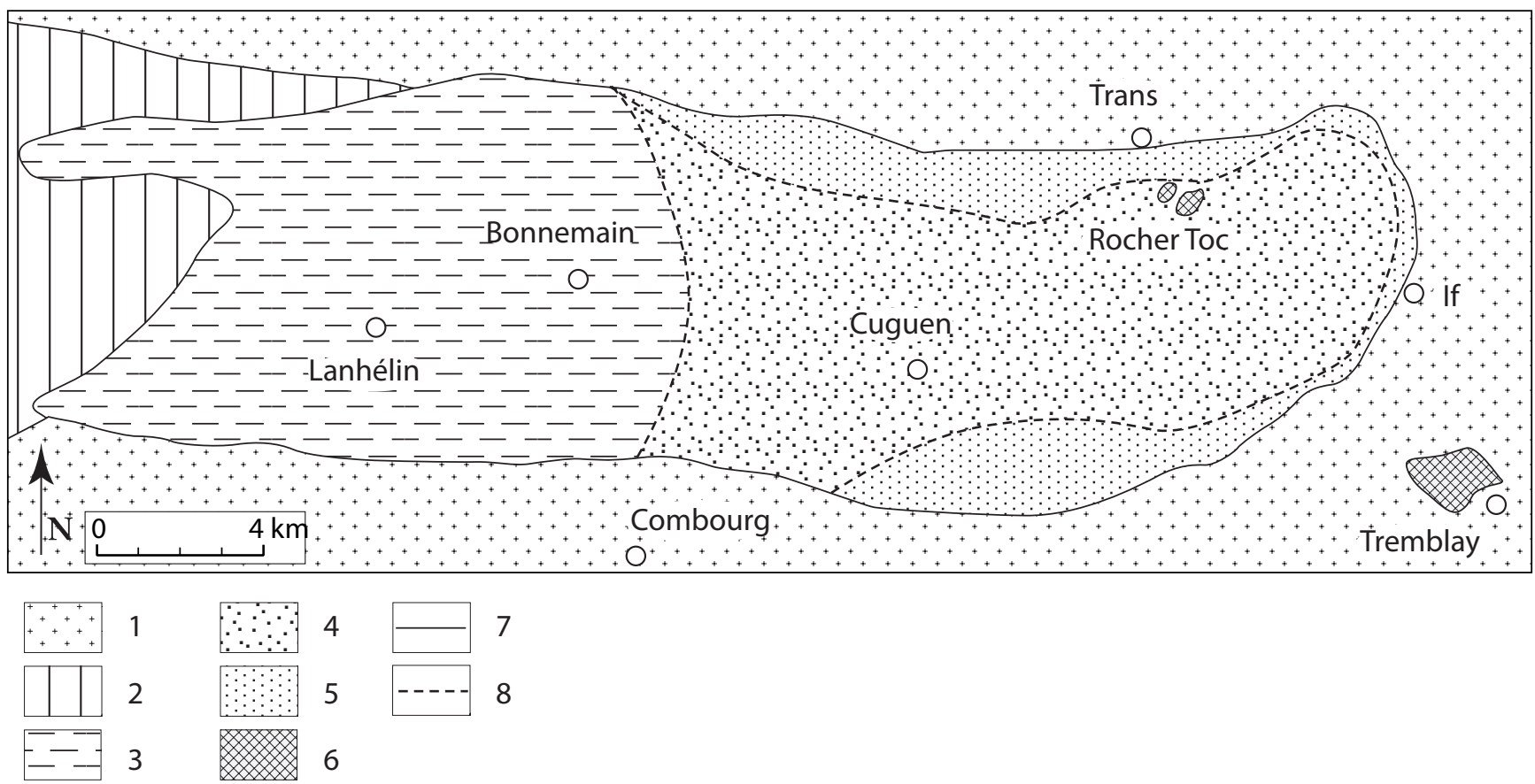

Figure 8 : Pluton composite de Lanhélin. D’après Jonin, 1969 et 1981. 1 : Briovérien (métamorphisé au contact du massif granitique; 2 : autres formations encaissantes; 3 : granite bleu de Lanhélin; 4 : granite gris de Cuguen; 5 : granite clair de La Fontenelle (lf); 6 : pointement leucogranitique; 7 : Contact tranché; 8 : contact (progressif?). Les filons de dolérite n’ont pas été figurés.

Figure 8: Lanhélin composite pluton. 1, Brioverian (metamorphozed at the contact of granite); 2, other host-rocks; 3, blue Lanhélin granite; 4, grey Cuguen granite; 5, clear La Fontenelle granite (lf); 6, leucogranitic outcrop; 7, clearcut contact; 8, other (progressive?) contact. Dolerite veins not reported.

Dès le Néolithique, les énormes blocs granitiques disséminés à la surface du sol ont suscité leur érection. Avec ses neuf mètres de haut, le menhir du Champ-Dolent est ici, sans conteste, le mégalithe le plus célèbre, déjà cité par Dubuisson-Aubenay en 1636, puis par Mérimée en 1836 dans ses " Notes de voyage ". Érigé sur des schistes briovériens recoupés par des filons doléritiques, son site d'extraction demeure énigmatique par suite de sa position à distance sensiblement égale entre les massifs de Lanhélin au sud et de Saint-Broladre à l'est. A priori, quand on connaît les masses énormes que peut livrer le premier pluton, une provenance méridionale parait la plus probable. (Le menhir de SaintMarcan cité plus haut n'atteint pas trois mètres de haut, ce qui reflète sans doute le débit du second de ces massifs et peut constituer une présomption a contrario quant à l'origine du Champ-Dolent). La même interrogation subsiste pour le menhir du " Marais du Mesnil " à Pleine-Fougères, emplacé lui aussi sur le Briovérien, à distance à peu près égale entre les deux massifs précités. Quoi qu'il en soit, le granite de Lanhélin a certainement livré des mégalithes ainsi que l'attestent le menhir christianisé de la " Pierre Longue » à l'ouest de Bazouges-La-Pérouse à sa bordure méridionale et le menhir de La Butte à Cuguen.

Les carrières ont été extrêmement nombreuses, souvent très rapprochées les unes des autres dans le faciès Lanhélin sensu stricto : La Chauffetière, L'Orientale, Le Rouvre, Bécanne... (fig. 9; Calvi, 1964, 1969; Gargi, 1971, 1979; anonyme, 1993, 2007; Cucarull, 2001). Plusieurs, encore en activité (entreprises Hignard, Socal...) sont aujourd'hui très profondes, livrant des "blocs dimensionnels ", extraits par les méthodes les plus modernes : machines de perforation hydraulique, minage très soigneusement dosé (aujourd'hui quatre fois moins chargé qu'il y a une vingtaine d'années) et transportés par de puissants chargeurs. L'exigence de la qualité a entraîné l'accumulation d'immenses tas de déblais, à présent récupérés pour des enrochements et pour l'obtention de granulats, valorisant ainsi l'ensemble de la production (anonyme, 2007).

Les modalités de l'extraction en profondeur sont en grande partie conditionnées par la disposition des diaclases ou fissures naturelles qui découpent le granite selon trois directions principales. Seules les carrières où la distance entre les diaclases est forte sont susceptibles de fournir de grands éléments. Dans les carrières de Lanhélin, les diaclases affec- 
Figure 9: Très forte densité des carrières abandonnées - ou encore en activité - aux environs de Lanhélin. D’après les données communiquées par Michel Minor, consultant.

Figure 9: Very high density of abandoned - or still active - quarries near Lanhélin. After data communicated by Michel Minor, consultant.

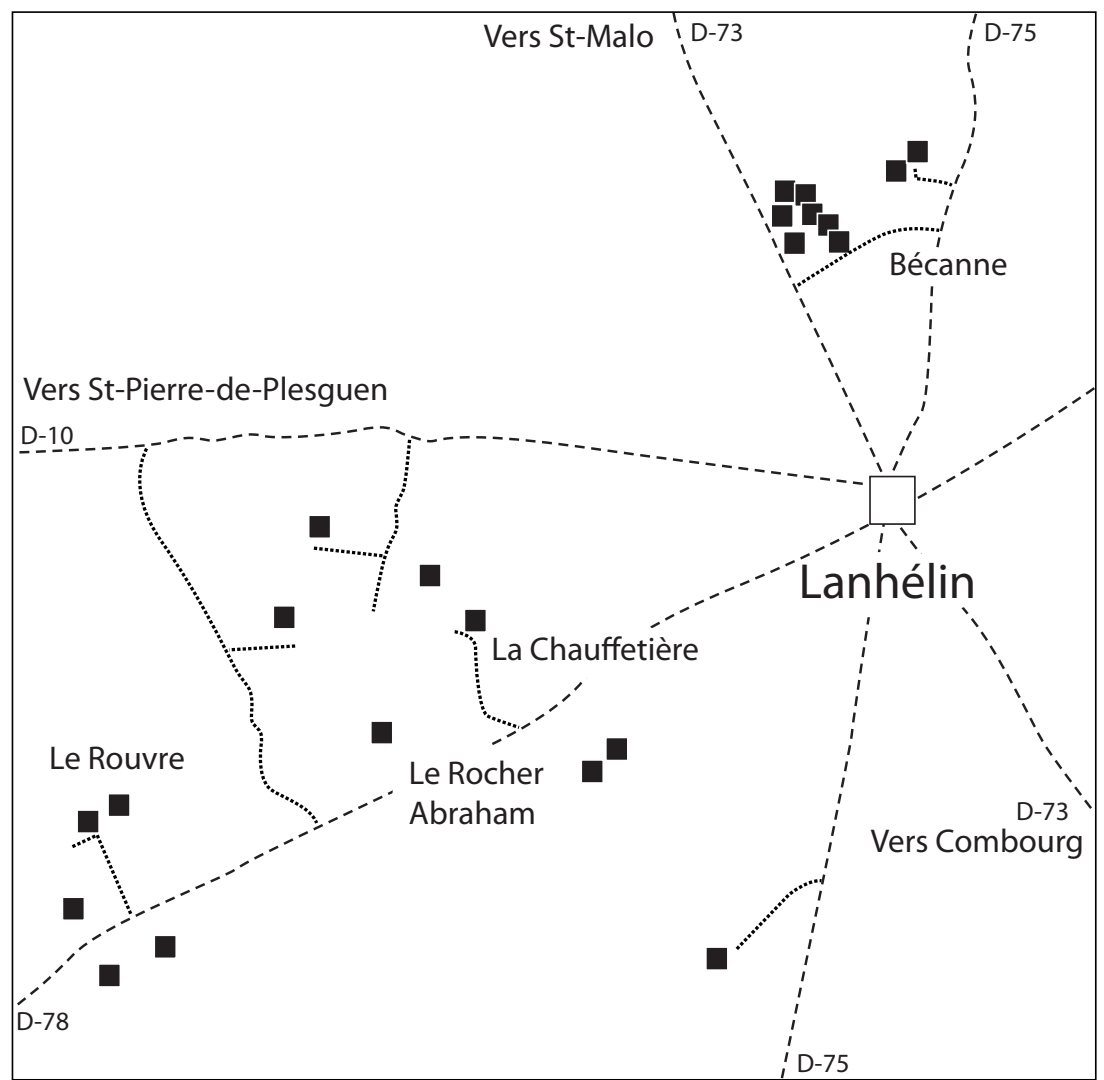

tent la disposition suivante : les fissures est-ouest et nord-sud sont subverticales; la troisième direction est subhorizontale. Ces directions correspondent respectivement à la «feuille ", à la « rogne " et à la « contrefeuille » ou " hubert » des carriers (Jonin, 1981). Plus précisément, la feuille est-ouest selon laquelle se fait le meilleur débitage de la roche correspond à une légère fluidalité liée à la mise en place du magma; la rogne nord-sud coïncide avec une importante direction de fracture, empruntée par les nombreux filons doléritiques (disposition bien visible dans la carrière Guillou-Granits).

Le transport du granite a longtemps posé de délicats problèmes, entraînant des litiges. Vers la fin des années 1840, les charrois acheminant les granites des carrières de Bécanne, de La Chauffetière et du Rouvre pour la chaussée de SaintMalo et son bassin à flot étaient tirés par vingt chevaux! Mais ces chargements pondéreux détérioraient les chemins qu'il fallait sans cesse "recharger ". Pour preuve, citons, parmi bien d'autres, les délibérations du conseil municipal de Saint-Pierre-de-Plesguen en octobre 1850. Selon les dires dudit conseil, l'ingénieur des Ponts et Chaussées fait transporter, depuis les carrières du Rouvre en SaintPierre-de-Plesguen et de Bécanne en Lanhélin, une énorme quantité de granite pour être employé au viaduc de Dinan. Lorsque les transports ont commencé, le chemin vicinal $\mathrm{n}^{\circ} 2$ venait d'être remis dans un état de viabilité complète sur une longueur de $3 \mathrm{~km}$; or, depuis - toujours selon le conseil municipal - « les transports ont déjà broyé la pierre et creusé de profondes ornières. Si les transports continuent pendant la saison pluvieuse, le chemin va être mis hors de service ». Dans ces conditions, ledit conseil s'estime en droit de formuler une demande d'indemnité. Dans sa réponse, l'ingénieur fait remarquer que ces carrières livrent des pierres pour toutes les constructions qui s'exécutent dans un certain rayon et pas exclusivement à la régie du viaduc de Dinan; en fait, les fournitures aux autres chantiers de la région sont beaucoup plus considérables (Chauris, 2002). Un cas original est fourni par l'utilisation du Couesnon, à une période imprécisée, pour le transport du granite de La Fontenelle (à l'extrémité orientale du massif de Lanhélin) avant son acheminement par mer jusqu'à Saint-Malo.

Les utilisations du granite bleu de Lanhélin, anciennes, prolongées et toujours intenses demanderaient de longs développements. En fait, à ce jour, aucun essai de dénombrements n'a encore été tenté; il nécessiterait des investigations approfondies tant dans les archives que sur le terrain. En voici un rapide survol.

Les édifices religieux concernés sont innombrables. À Saint-Malo, dans la cathédrale Saint-Vincent, la partie médiane de la façade occidentale remontant à la seconde partie du XviII ${ }^{e}$ siècle est en granite de Lanhélin superbe- 
ment appareillé; la tour massive de l'église de Saint-Servan (vers 1840) a mis en œuvre la même roche. Dans l'église de Lanhélin, on admirera quatre magnifiques colonnes monolithes en pierre locale. À Pleuhiden-sur-Rance, ce même granite a été utilisé pour le soubassement, les escaliers d'accès à la porte latérale nord... ; à Saint-Hélen, on le trouve dans le portail de la façade du clocher... ; à Paramé, il est utilisé pour le puissant soubassement du calvaire érigé près de la chaussée du Sillon; dans l'île Tristan, en face de Douarnenez (Finistère), on y a recouru pour une chapelle...

La confection des monuments funéraires est actuellement l'une des principales utilisations du granite de Lanhélin. Il n'est sans doute à présent aucun cimetière dans l'ouest de la France qui ne possède une ou plusieurs tombes en cette roche dont la sombre tonalité bleutée est renforcée par l'excellence du poli. En fait, dans ce domaine, sa diffusion paraît bien s'étendre à l'ensemble de la France : ne l'avons-nous pas noté aussi bien dans de grandes métropoles (Paris, Lille, Rouen, Nantes, Rennes, Bordeaux, Toulouse...) que dans de nombreuses petites villes? Mais cet engouement pour l'art funéraire ne date pas d'aujourd'hui. Dans le cimetière ombragé de Saint-Servan, de vieilles tombes apparaissent à la fois comme un hommage aux disparus et un témoignage des qualités du granite qui perpétue leur souvenir. Faut-il rappeler que le tombeau de Châteaubriand au Grand Bé, au large de Saint-Malo, est aussi en granite de Lanhélin (mais avec dix bornes-limites en granite de Brusvily, issu du massif hercynien de Dinan).

Un volume pourrait être consacré aux monuments aux morts en granite de Lanhélin érigés après la Grande Guerre, plus rarement après la dernière guerre, non seulement à proximité des zones d'extraction, mais aussi bien au-delà, attestant ainsi la célébrité du matériau. Le type le plus répandu est - comme partout - la stèle pyramidale à quatre pans dressée sur un puissant socle (Lanvalay, La Boussaine...); la stèle peut être plate (Trévérien); deux piliers peuvent encadrer une large plaque médiane (Les Champs-Géraux); parfois, le granite de Lanhélin est réservé aux plaques nominatives (Batz). Plus original est le monument de Lancieux dont la partie basale présente un soldat couché.

Dans l'habitat, le granite de Lanhélin peut être réservé à la pierre de taille. Il en est ainsi à Mordreux, en Pleudihensur-Rance, les moellons étant en granite de la Rance. Au château de Kerrozen, en Taden, le granite de Lanhélin est admirablement façonné.

Ouvrages défensifs : malgré la concurrence du granite de Chausey, Saint-Malo a fait un large appel au granite de Lanhélin (Fort-National, fort du Naye...). Plus au sud, le fort Saint-Père, près de Châteauneuf d'Ille-et-Vilaine, a recherché ce même matériau.
Travaux publics : dans le viaduc routier de Dinan, le granite de Lanhélin est associé à d'autres granites (Chauris, 2002). La même pierre a été utilisée à Saint-Malo, entre autres pour le parapet du Sillon, mais aussi pour le phare des Bas-Sablons allumé en 1868 (Chauris, 2011) ou pour la tablette du quai dans le port de Dinan... Le granite de la carrière de La Chauffetière en Saint-Pierre-de-Plesguen a été recherché pour l'écluse du Châtelier. Des bittes d'amarrage ont été aussi façonnées dans le granite de Lanhélin. Les bases des piles du viaduc de Lessard sur la Rance ont mis en œuvre le même granite en grand appareil (Chauris, sous presse c).

Dans les aménagements urbains, le granite de Lanhélin est à présent très recherché. Ici aussi, les exemples sont fort nombreux. À Rennes, citons la fontaine placée à l'entrée de la rue de Coëtquen; à Lesconil, dans le sud-Finistère, un beau dallage devant le port... Paris occupe une place tout à fait remarquable : dallages, bordures et entourages d'arbres sur les Champs-Élysées; place Stravinsky, ce sont des dallages en grands éléments; dallages encore dans la Cour Napoléon au Louvre et place de l'Hôtel-de-Ville, ici en association avec le granite rouge de La Clarté en Ploumanac'h et le granite " Gris celtique » de Plounevez-Quintin... Au total, les dallages en granite de Lanhélin couvrent à Paris des milliers de mètres carrés. Toujours à Paris, plusieurs bâtiments ont fait appel au même granite : façade de la Cité des Sciences-La Villette; Opéra-Bastille; le siège de Canal Plus, 5 rue Couchy, a mis en œuvre 5000 mètres carrés de dallage et d'aménagements extérieurs. Le réaménagement du port de La Bourdonnais a demandé 2000 mètres carrés de granite flammé. Des bornes cylindriques ont été façonnées pour la place Stravinsky. Les bordures des bassins de la Cour Napoléon au Louvre soulignent l'aptitude du granite de Lanhélin au polissage. Le "Jardin de l'Atlantique ", audessus de la gare Montparnasse, a recherché la même pierre pour des motifs décoratifs sous forme de hautes plaques polies. Quelques monuments doivent aussi être évoqués : la haute stèle monolithe à section rectangulaire avec traces des trous de perforation disposés horizontalement des quatre côtés, servant de socle à la statue en bronze de Max Ernst (1891-1976) et surtout l'étonnante sphère de deux mètres de diamètre, adoucie par la "Générale du Granit ", érigée dans la Cour d'Honneur du palais de l'Assemblée nationale; il a été calculé que seul un vent de plus de $300 \mathrm{~km} / \mathrm{heure}$ pourrait en menacer l'équilibre.

Plusieurs monuments commémoratifs ont également fait appel au granite de Lanhélin. Dans le passé, ce furent les belles colonnes monolithes cylindriques de l'édifice érigé au "Champ des Martyrs " près d'Auray. Beaucoup plus récemment, citons le monument élevé à Paimpol «à la gloire de la Marine marchande de la France libre " près de l'entrée du port ou, à Guiliers, le monument aux déportés... 
Bien d'autres exemples d'utilisation du granite de Lanhélin pourraient encore être évoqués, soulignant les aptitudes multiformes de cette roche au façonnement : vers l'extrémité de l'île Callot, en baie de Morlaix, une table d'orientation; à Grenoble, le monument des Jeux olympiques. Dans les symposiums estivaux consacrés à la sculpture sur granites bretons, le Bleu de Lanhélin occupe une place de choix (anonyme, 1993) : parmi d'autres, citons « Le Tricot de la Terre " par Harada; "Travers-Traverse-Passage " par Schewzuck; saint Malo sculpté en 2009 par Patrice Le Guen et aujourd'hui dans la Vallée des Saints à Carnoët (Côtesd'Armor)... Et, à peine croyable, une barque monolithe de

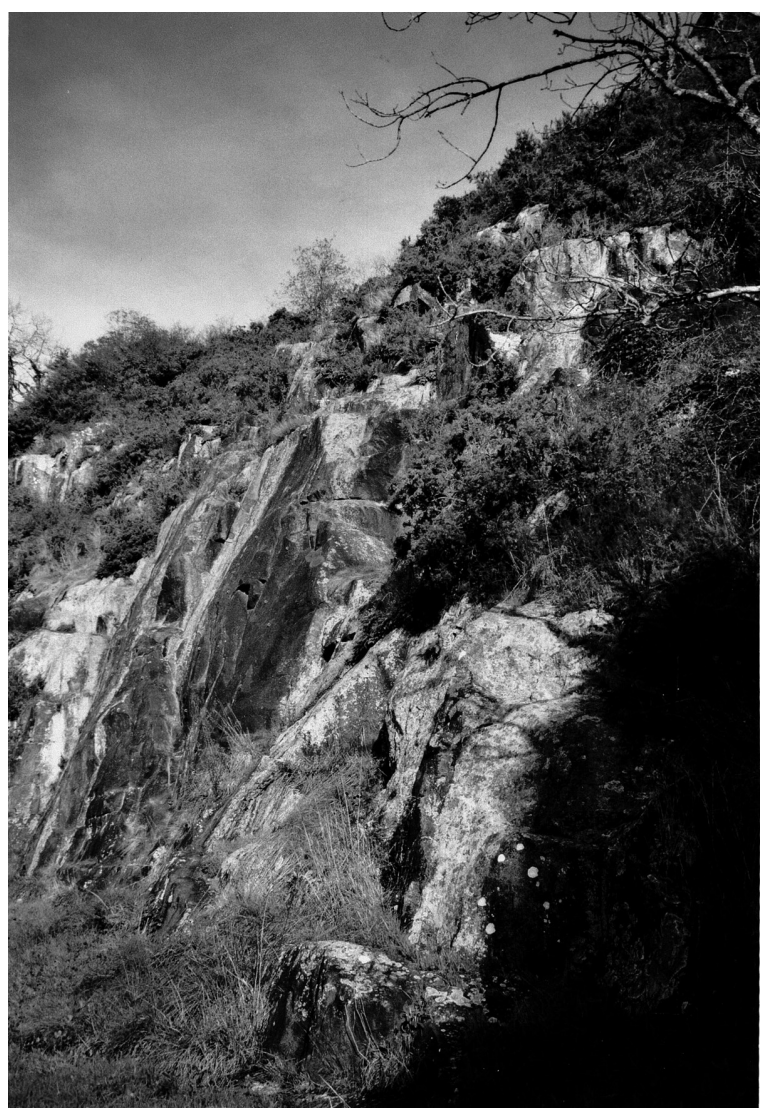

Photo I : Au Mont-Dol, le front de taille d'une des anciennes carrières de granite est peu à peu envahi par la végétation (20/10/2012).

Photo I: Le Mont-Dol: front of one of the ancient granite quarries progressively invaded by vegetation. quatre mètres de long - et qui flotte - façonnée pour célébrer l'an 2000 par le sculpteur Jean-Yves Menez à partir d'un bloc de 33 tonnes extrait dans la carrière de La Socal!

Achevons ce trop bref aperçu sur l'utilisation du granite de Lanhélin par quelques exemples de sa mise en œuvre à l'étranger (collectif, 1998), attestant ainsi sa renommée internationale : façades de la banque de Chine à Hong Kong; façade de l'Empire Tower à Djakarta; façade d'une tour à Houston; sol et fontaine du musée d'art moderne à Tokyo; gare de Kyoto où nous avons eu la joie et la surprise de l'identifier...

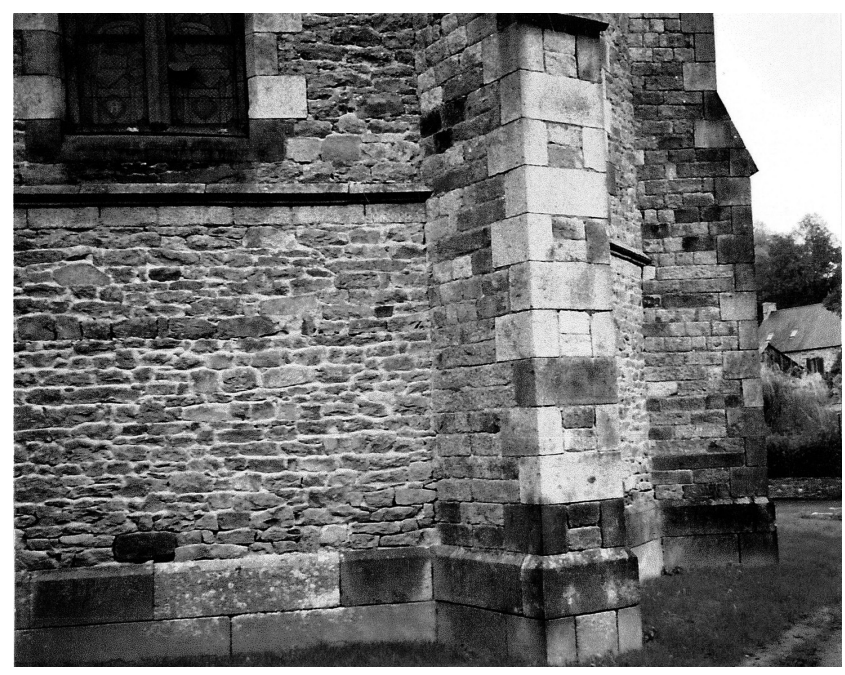

Photo II : Église de Saint-Broladre. Appel au granite local, tant pour la pierre de taille que pour les moellons (28/10/2012). Photo II: St Broladre church: use of local granite, as well for freestones as for rubble. 


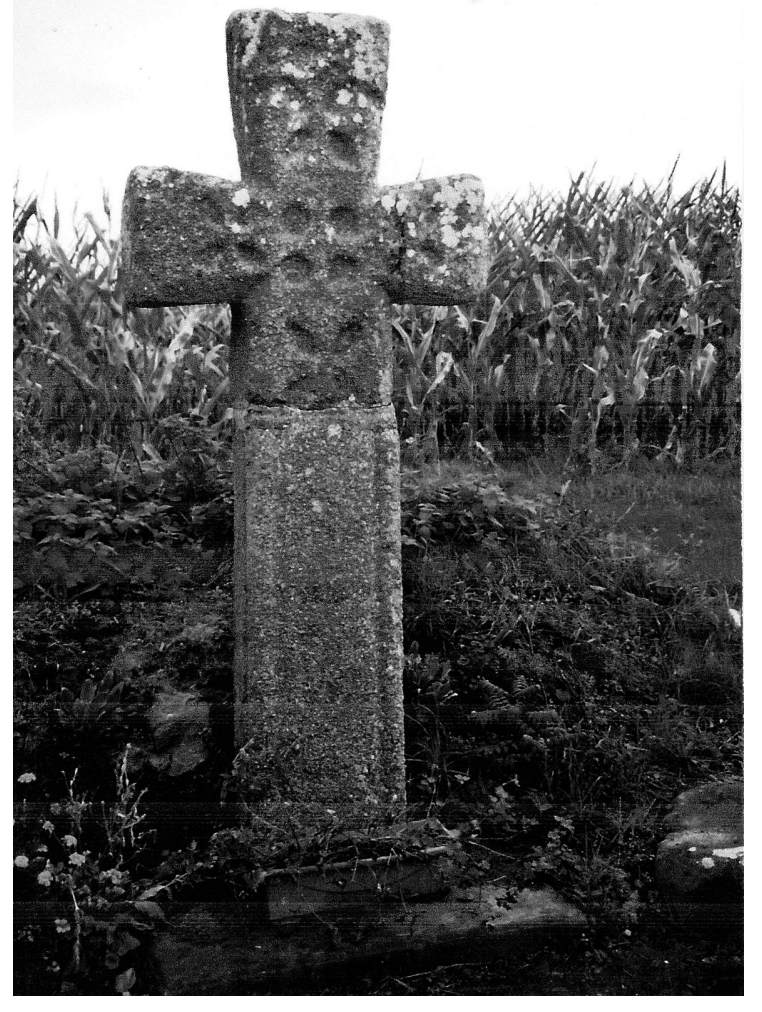

Photo III : "Croix du Seigneur " à Saint-Marcan en granite de Saint-Broladre. (28/10/2012).

Photo III: "Lord's Cross" at St Marcan, of St Broladre granite.

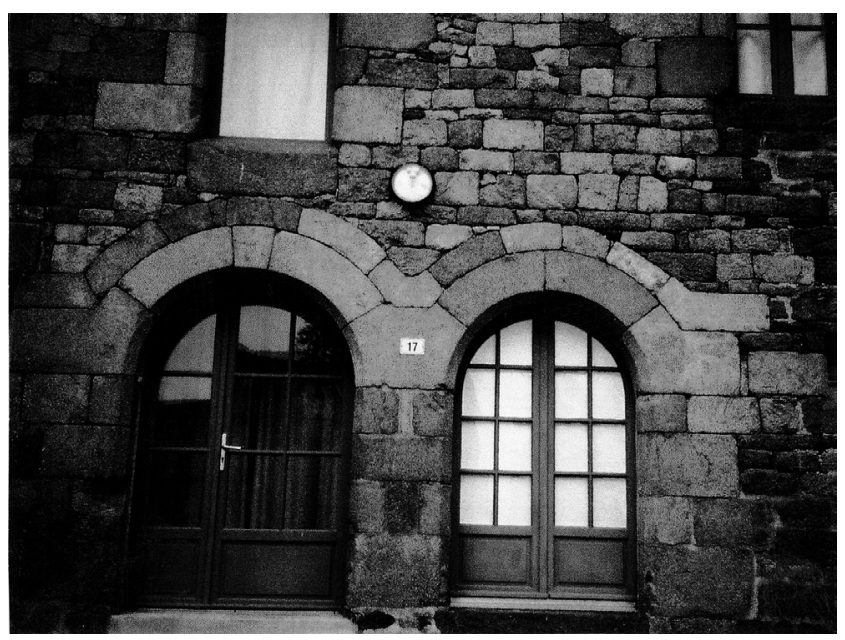

Photo IV : Au bourg de Dingé. Demeure à portes géminées soulignant l'aptitude du granite local au façonnement élaboré. Emploi aléatoire du faciès bleu (sain) et du faciès beige (altéré) (10/11/2009).

Photo IV: In the village of Dingé, residence with geminated doors underlining the capacity of local granite to be elaborately fashioned; random use of blue facies (fresh) and beige one (weathered).

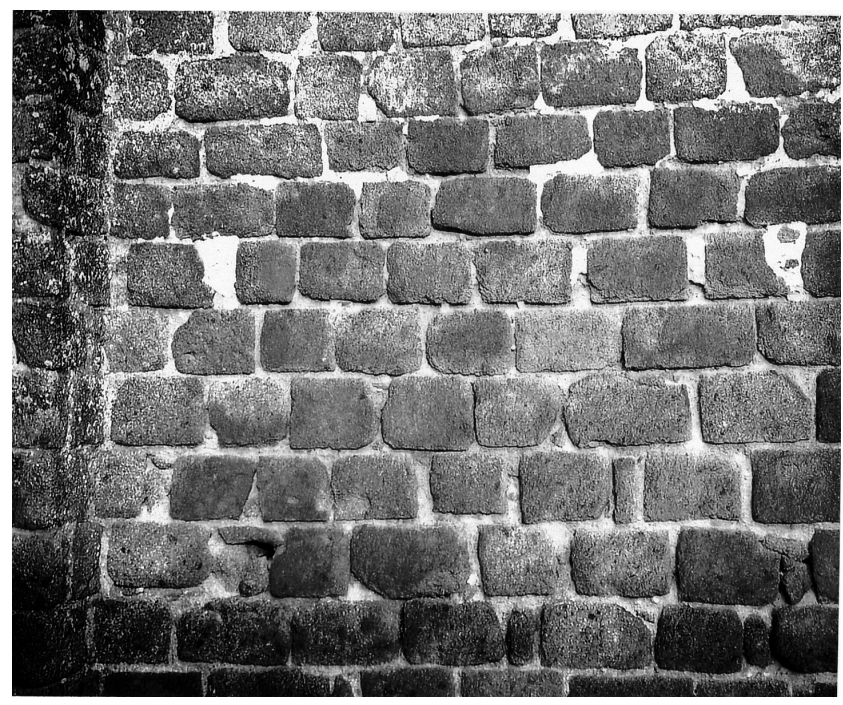

Photo V : Église de Hédé. Élévation occidentale en moellons régulièrement assisés, façonnés dans le granite brunâtre local (07/10/2005).

Photo V: Hédé church: western elevation of regularly layed rubble, local brownish granite.

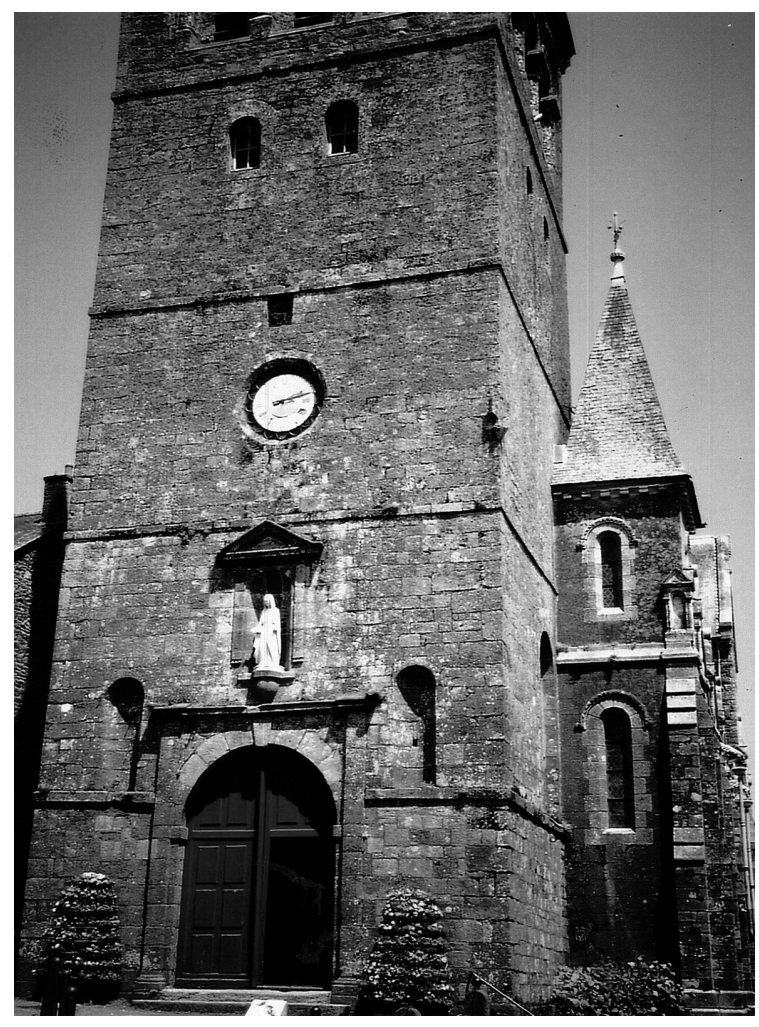

Photo VI : Église de Bécherel, polyphasée et polylithique (04/06/2009).

Photo VI: Bécherel church, polyphazed and polylithic. 


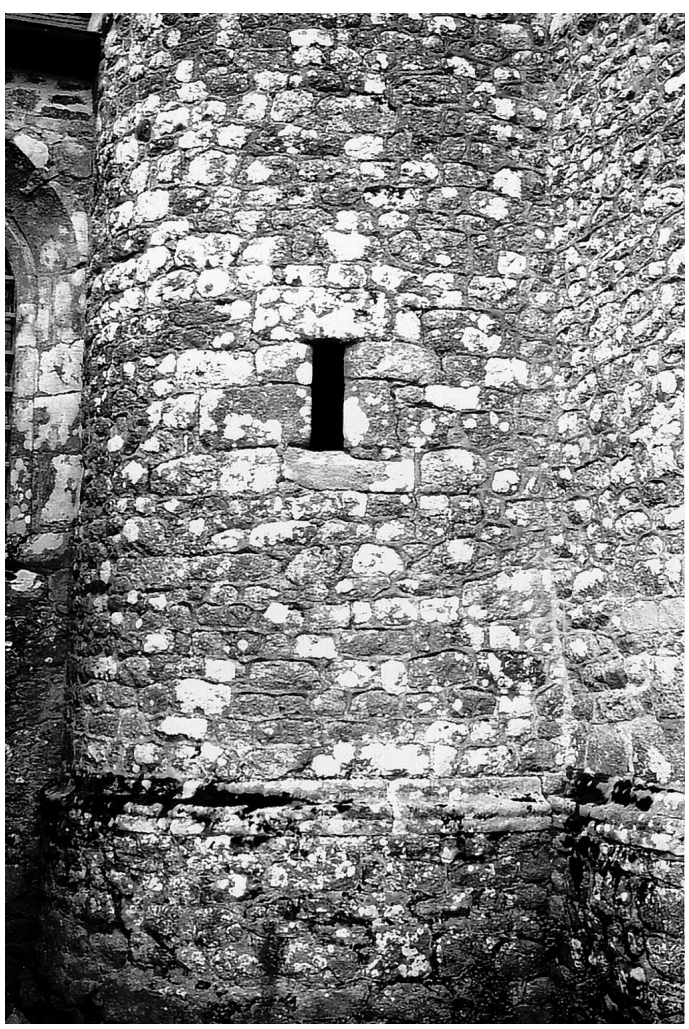

Photo VII : Église de Miniac-sous-Bécherel : tourelle du clocher en moellons granitiques de Bécherel et cordon en granite de Languédias (07/10/2005).

Photo VII: Church of Miniac-sous-Bécherel: tourelle of the bell-tower in Becherel granite rubble; cordon of Languedias granite.

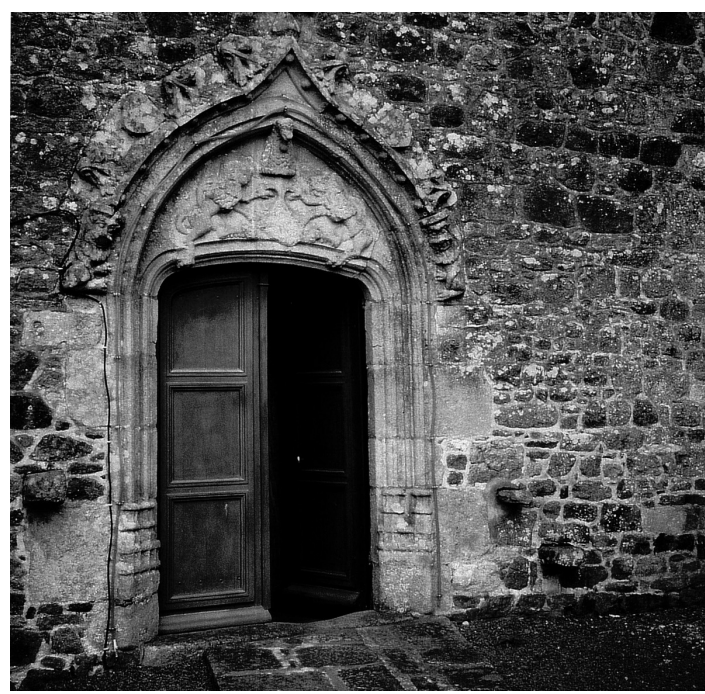

Photo VIII : Église de Miniac-sous-Bécherel : porte occidentale en clair granite de Languédias en fort contraste avec l'élévation en granite brunâtre de Bécherel (07/10/2005).

Photo VIII: Church of Miniac-sous-Bécherel: western door of clear Languedias granite, in strong contrast with elevation in brownish Bécherel granite.

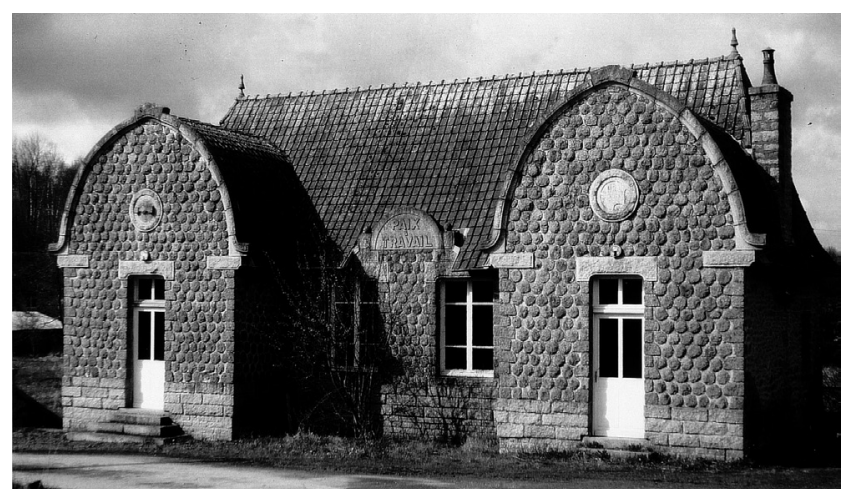

Photo IX : Bâtiment de l'ancienne coopérative "L'Avenir " (Louvigné-du-Désert) inauguré en 1946 (16/03/1994).

Photo IX: Building of the ancient "L'Avenir" cooperative at Louvignédu-Désert, inaugurated in 1946.

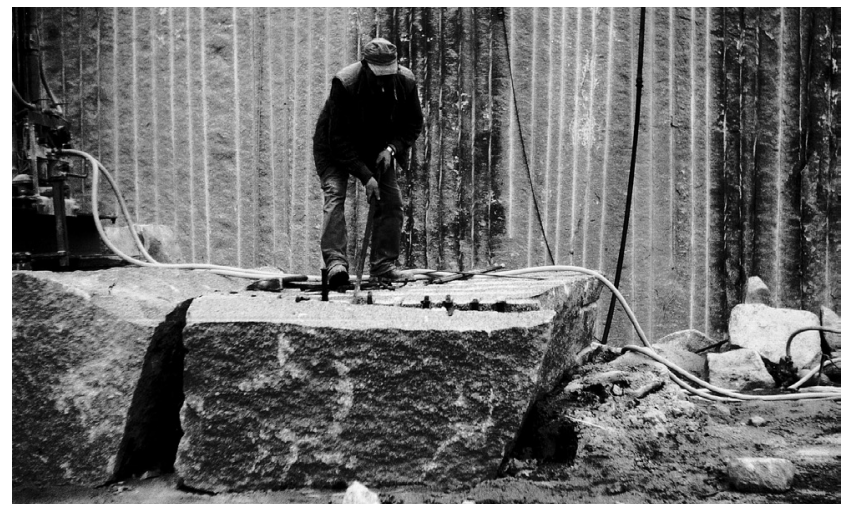

Photo X : Carrière de La Piochais à Bazouges-du-Désert. À l'arrière-plan, "masse primaire " découpée par des trous de perforation verticaux. Au premier plan, fente pour l'obtention de «blocs dimensionnels " par la méthode des coins (16/09/1994).

Photo X: La Piochais quarry at Bazouges-du-Désert. In background, "primary mass" of granite, cut by vertical perforation holes; in foreground, wedges cleaving to obtain "dimensional blocks".

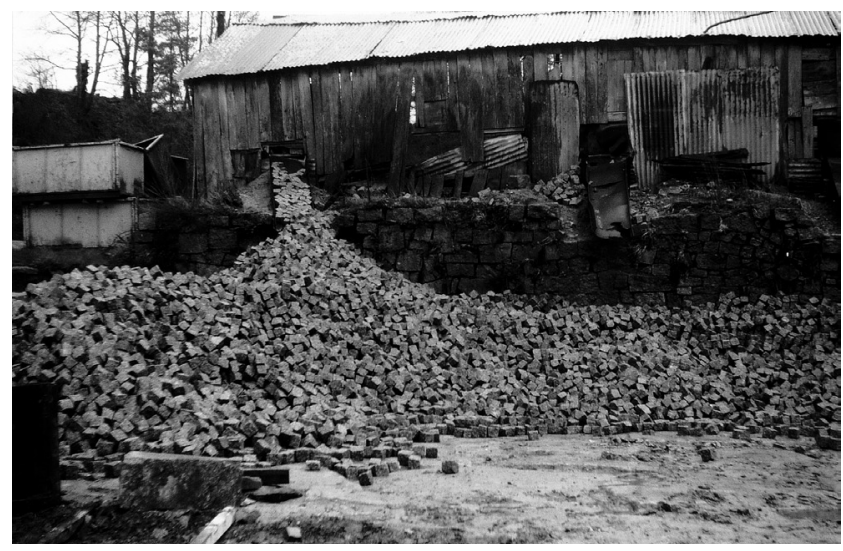

Photo XI : Carrière de La Morinais. Production de pavés (à la cliveuse) (16/03/1994).

Photo XI: La Morinais quarry: paving stones production with a splitting machine. 


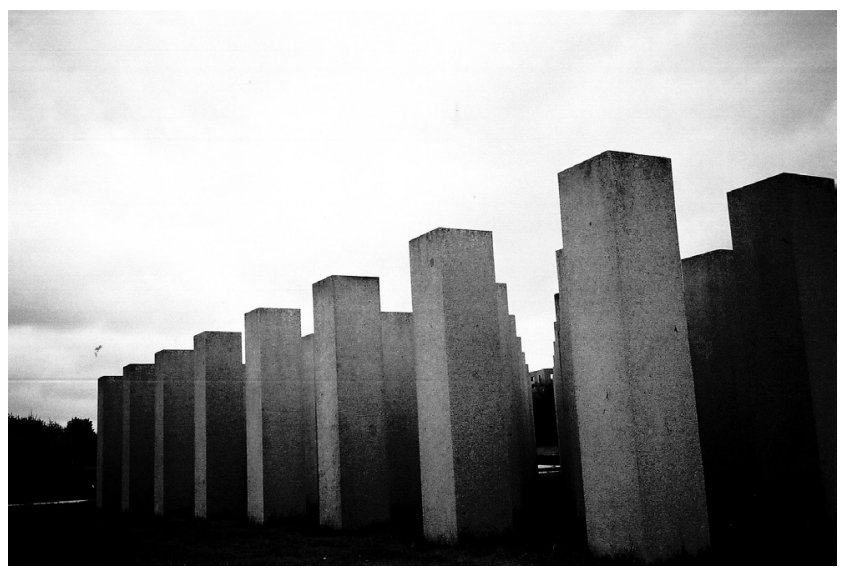

Photo XII : Vue partielle des soixante-douze colonnes en granite de la carrière Godard à Louvigné, dressées à Rennes sur le site de Beauregard.

Photo XII: Partial view of the 72 columns of granite from the Godard quarry at Louvigné, erected at Beau regard in Rennes.

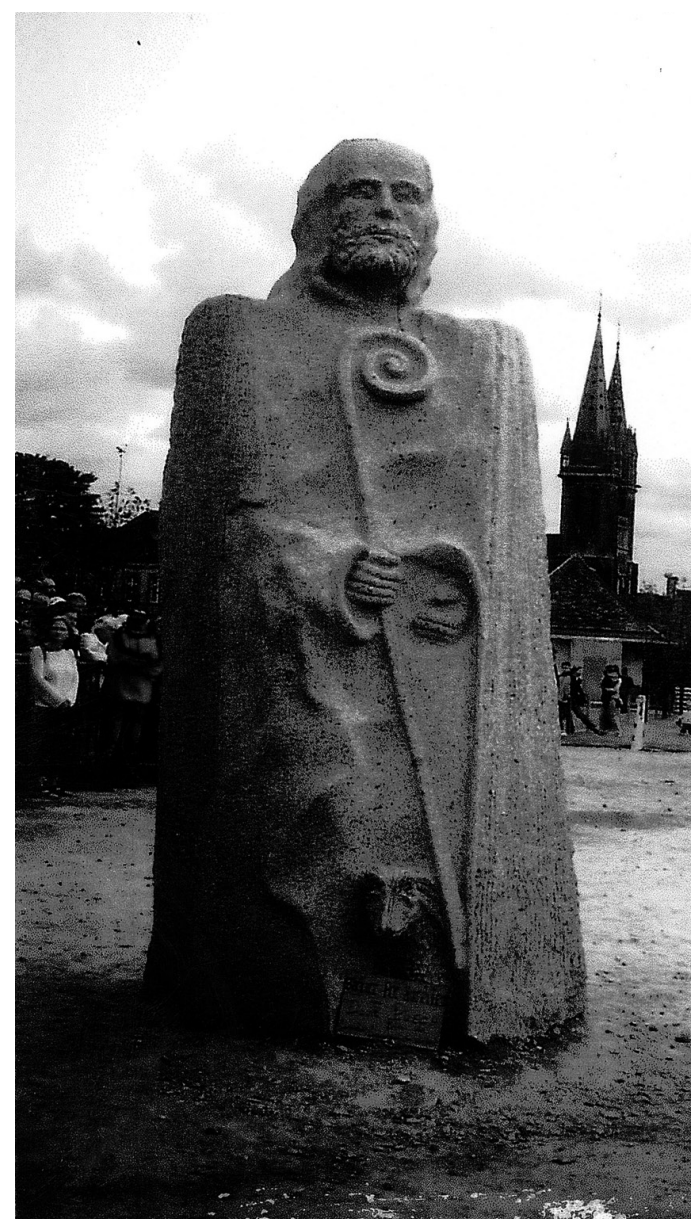

Photo XIII : Statue de saint Pol, sculpté dans le granite de Louvignédu-Désert en 2009 à Saint-Pol-de-Léon par David Puech.

Photo XIII: St Pol statue, sculptured in Louvigné-du-Désert granite at Saint-Pol-de-Léon by David Puech.

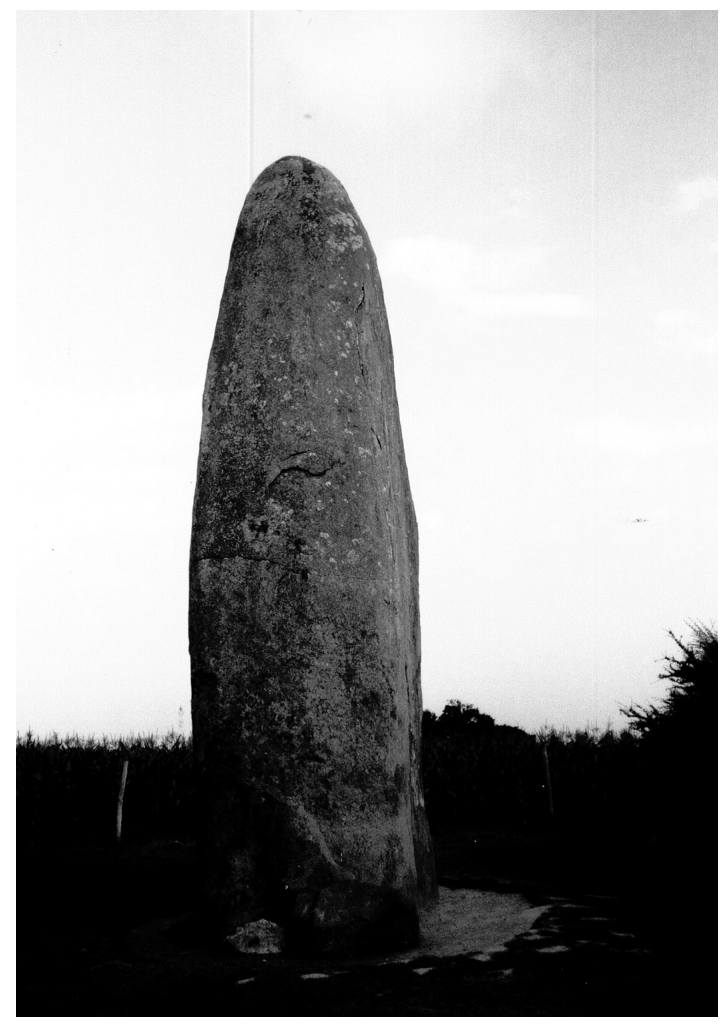

Photo XIV : L'érection du menhir du Champ-Dolent près de Dol-de-Bretagne a nécessité un transport de plusieurs kilomètres (30/08/2000).

Photo XIV: The Champ-Dolent menhir near Dol-de Bretagne: its erection needed a transport over several kilometres.

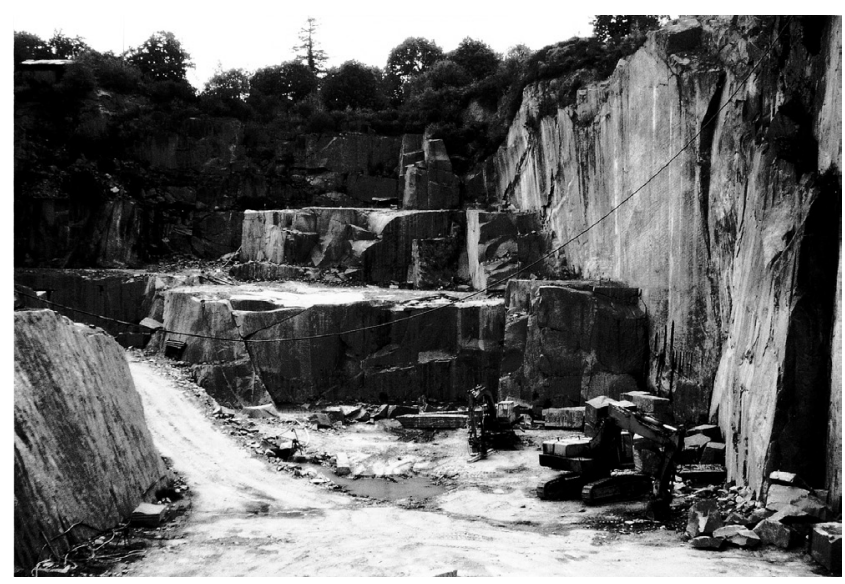

Photo XV : Vue partielle de la carrière Hignard extrayant le granite bleu de Lanhélin (24/08/1991).

Photo XV: Partial view of Hignard quarry, exploiting blue Lanhélin granite. 


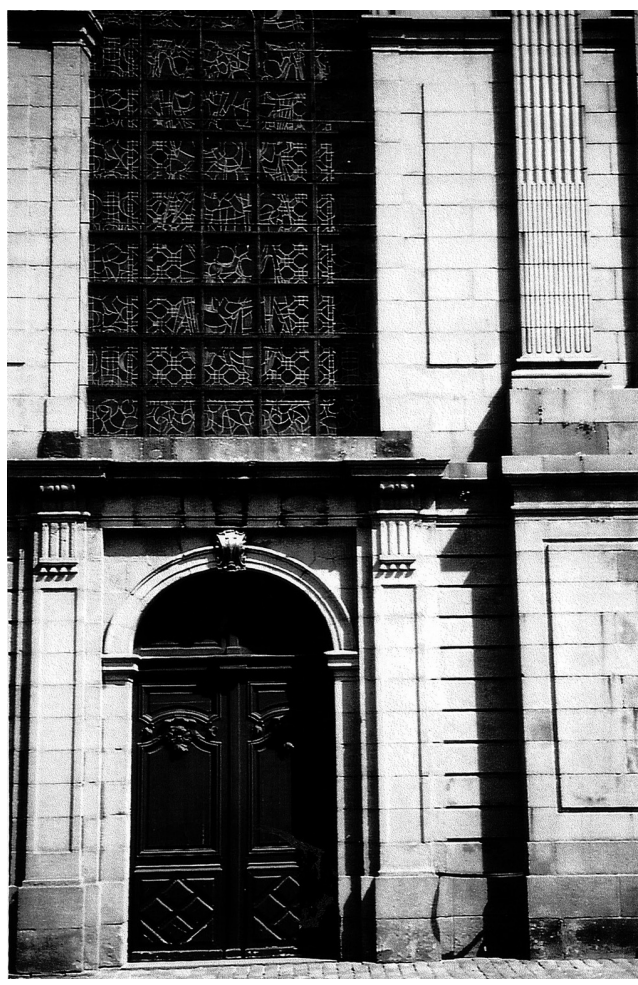

Photo XVI : Cathédrale Saint-Vincent à Saint-Malo. Partie médiane (seconde moitié du XVIII ${ }^{\mathrm{e}}$ siècle) de la façade occidentale, en granite de Lanhélin superbement appareillé (06/05/2010). Photo XVI: St Vincent cathedral, Saint-Malo. Median part (2 $2^{\text {nd }}$ half of $18^{\text {th }}$ century) of western façade: superbly bonded Lanhélin granite.

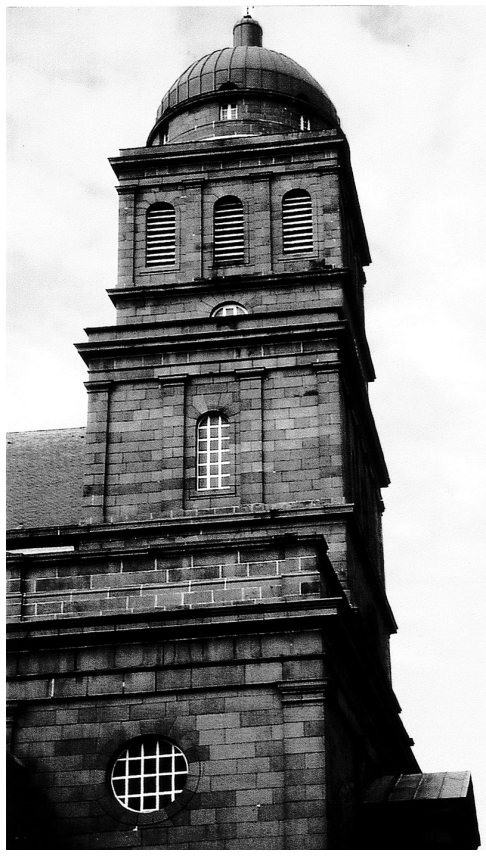

Photo XVII : Tour de l'église de Saint-Servan en granite de Lanhélin $(08 / 05 / 2010)$.

Photo XVII: Tower of Saint-Servan church, in Lanhélin granite.

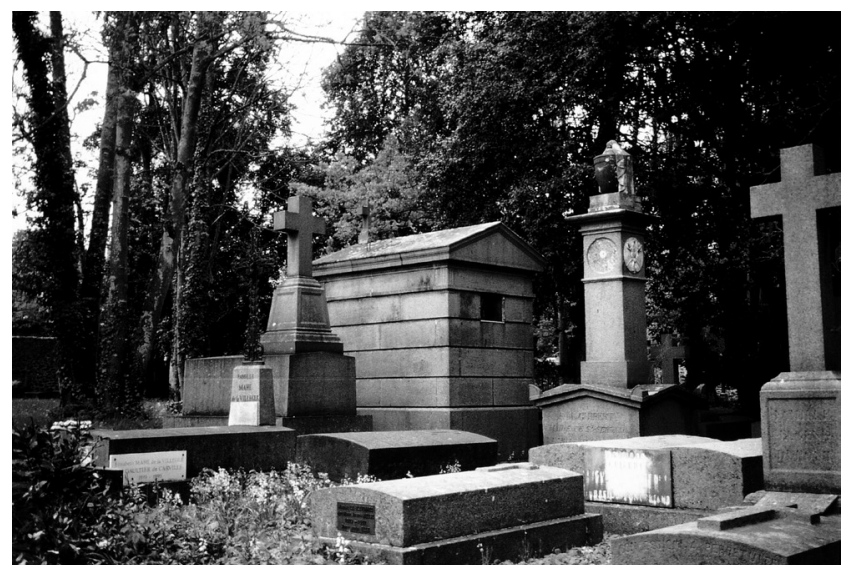

Photo XVIII : Au cimetière de Saint-Servan, noyé dans la verdure, se tient le festival silencieux du granite de Lanhélin (08/05/2010). Photo XVIII: In Saint-Servan graveyard, silent festival of Lanhélin granite sunk in greenery.

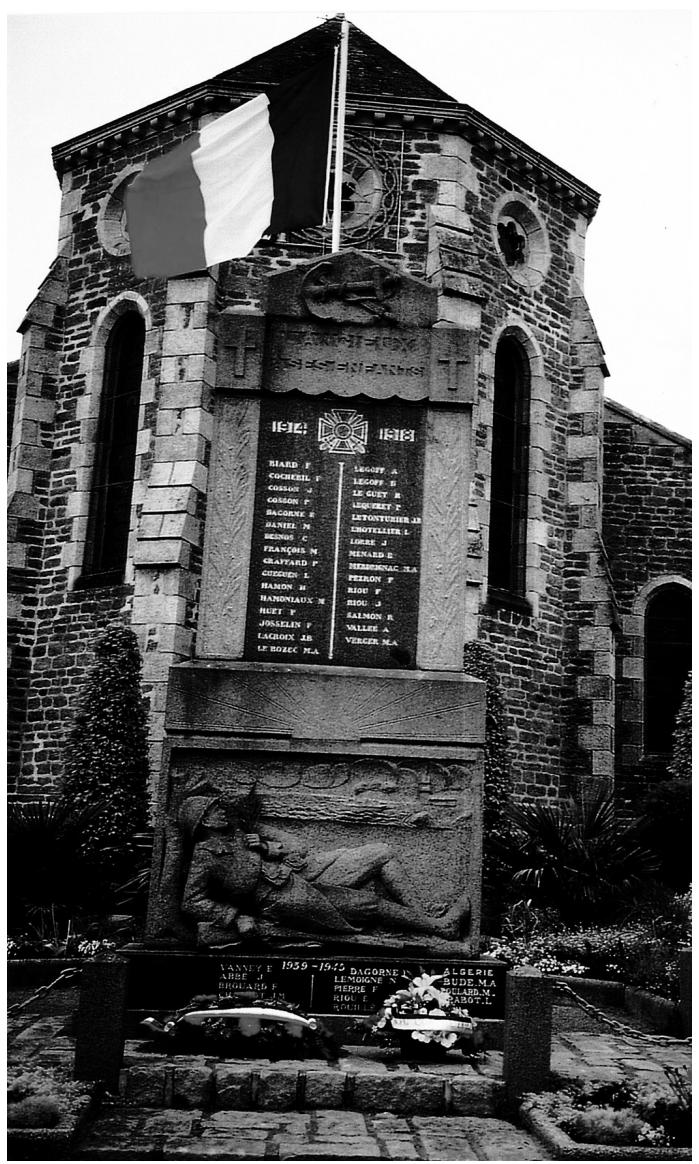

Photo XIX : Monument aux morts de Lancieux soulignant les aptitudes du granite de Lanhélin à la sculpture (08/05/2010). Photo XIX: The Lancieux war memorial emphasizes the ability of Lanhélin granite to be sculptured. 


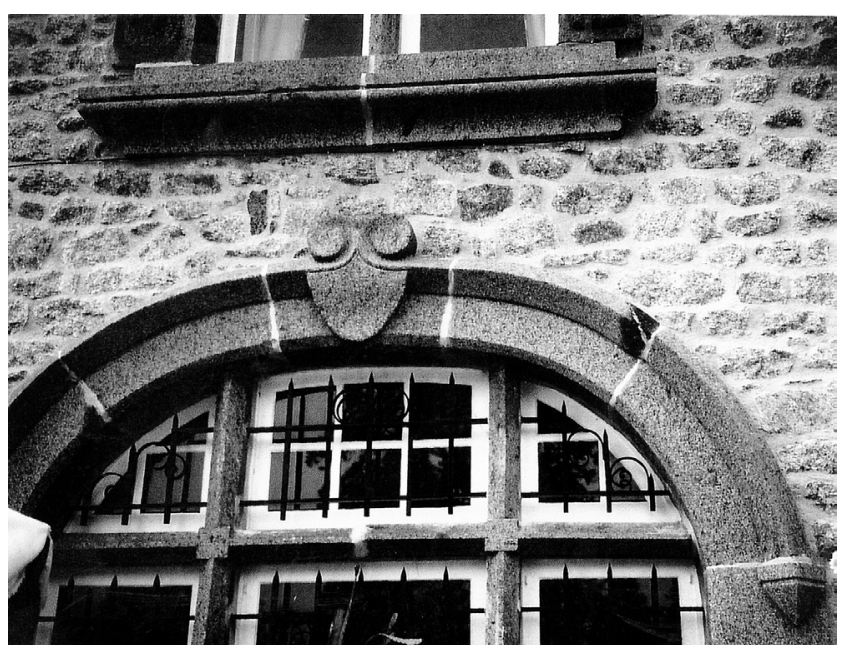

Photo XX : Mise en œuvre du granite de Lanhélin dans le château de Kerrosen en Taden (26/06/2005).

Photo XX: Use of Lanhélin granite in Kerrosen castle, commune of Taden.

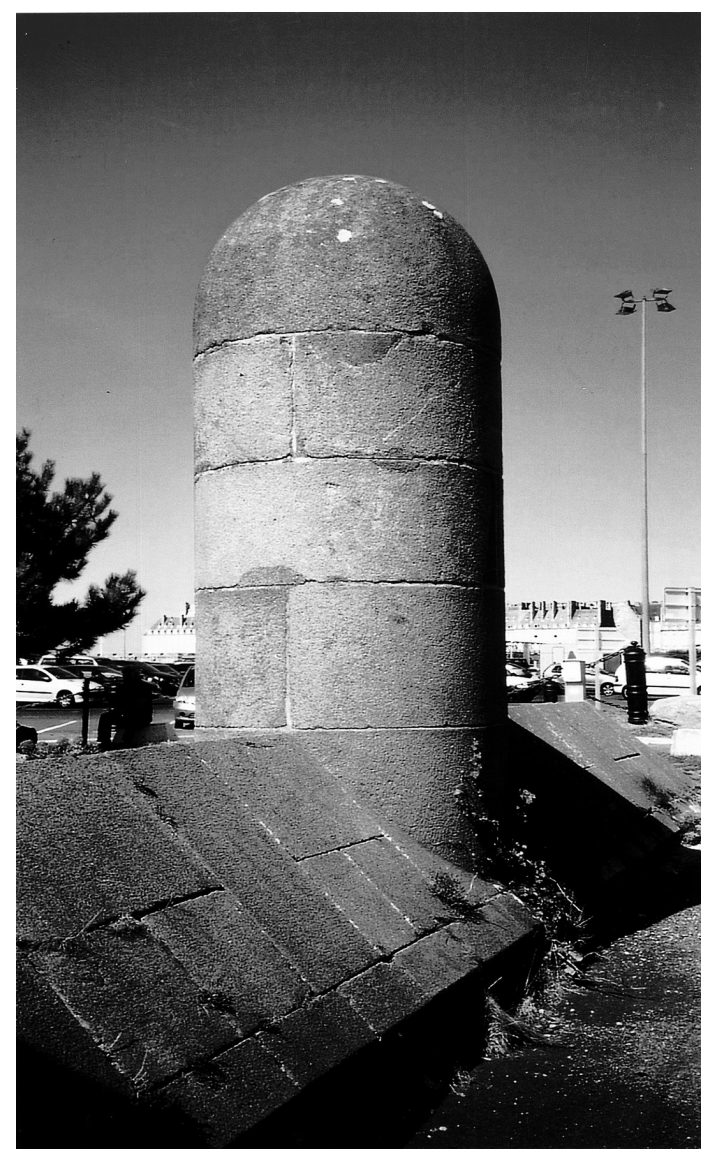

Photo XXI : Fort de La Naye à Saint-Malo. Mise en œuvre somptueuse du granite de Lanhélin (08/09/2010).

Photo XXI: La Naye fort, at Saint-Malo : lavish use of Lanhélin granite.

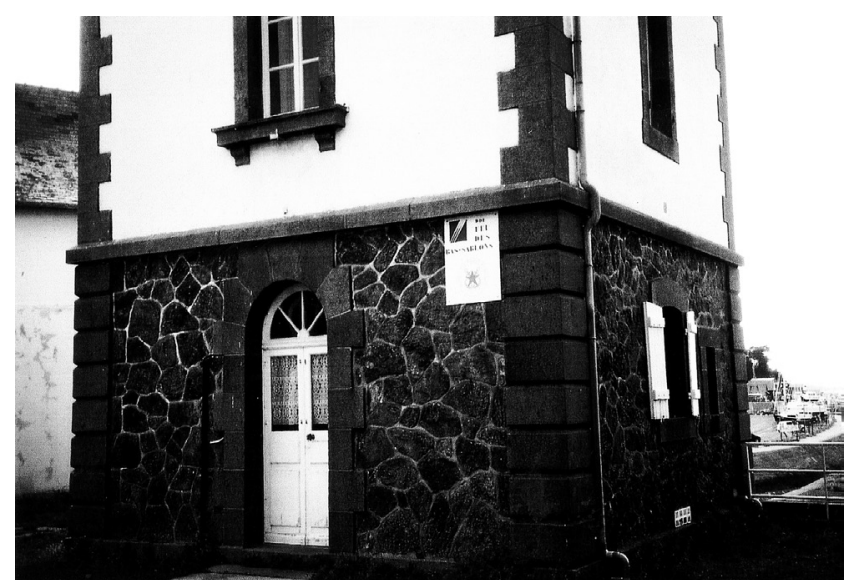

Photo XXII : Phare des Bas-Sablons à Saint-Malo. Chaînage d'angle et encadrement des ouvertures en granite de Lanhélin; bel appareil polygonal en granite migmatitique de provenance proximale (07/06/2010).

Photo XXII: Bas-Sablons lighthouse, Saint-Malo : angle clamping and doors framing in Lanhélin granite and fine polygonal bond of sublocal migmatitic granite.

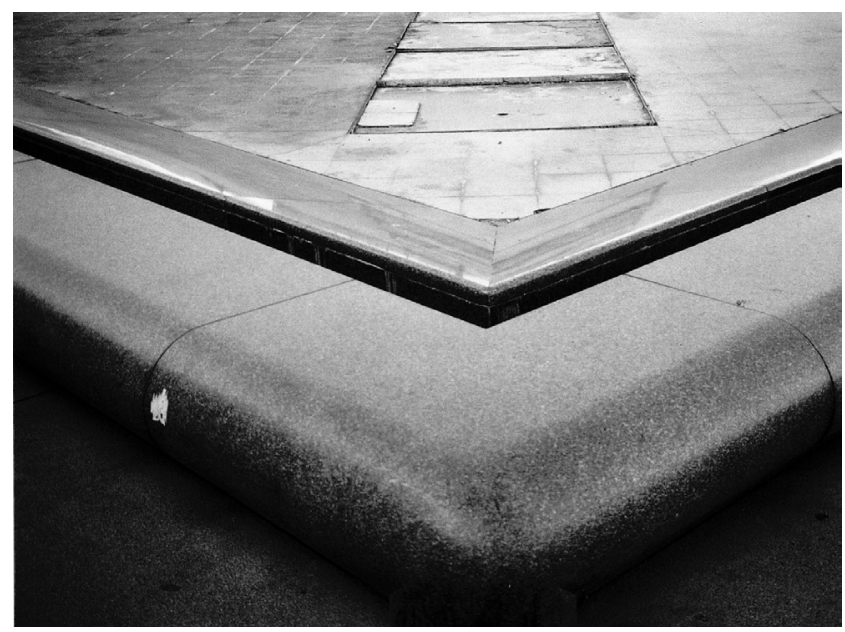

Photo XXIII : Paris, cour du Louvre. Bordure du bassin en granite de Lanhélin poli (11/10/2005).

Photo XXIII: Paris, Le Louvre courtyard: pond's edge in polished Lanhélin granite. 


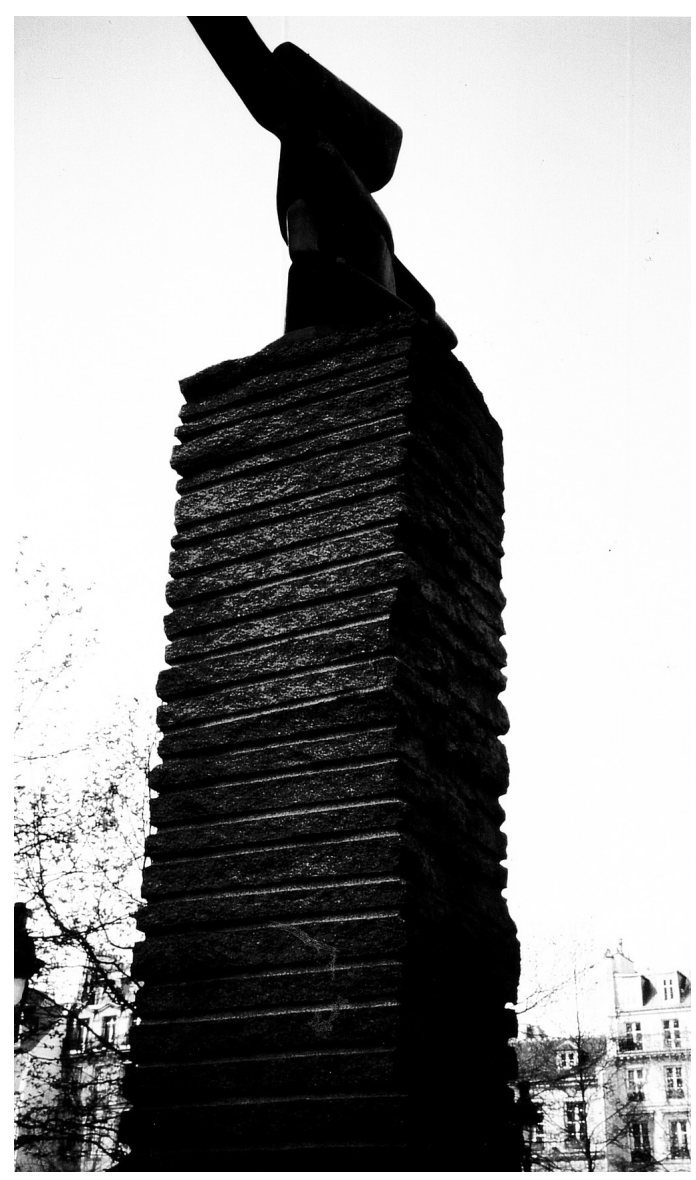

Photo XXIV : À Paris, socle en granite de Lanhélin de la statue de Max Ernst (04/04/2002).

Photo XXIV: In Paris, pedestal of Max Ernst statue, in Lanhélin granite.

\section{Remerciements}

Notre vive reconnaissance à Charles-Tanguy Le Roux pour la lecture pertinente du manuscrit.

\section{Bibliographie}

Andrieux J.-Y. (coord.), 2011 - Arthur Regnault, architecte (18391932). La quintessence de l'art sacré, Rennes, Presses universitaires de Rennes, $256 \mathrm{p}$.

Anonyme, 1993 - « Pour une renaissance de la sculpture sur granit breton ", Le Mausolée, 1993 (12), p. 74-79.

Anonyme, 2003 - «À Louvigné-du-Désert. Métamorphose d'une place ", Pierre Actual, n 805, p. 79-81.

Anonyme, 2007 - «À Lanhélin, la Socal valorise l'ensemble de sa ressource ", Pierre Actual, no 853, p. 48-51.

Calvi M., 1964 - « Voyage des granitiers allemands en France », Le Mausolée, 1964 (6), p. 1053-1085.
CALvi M., 1969 - «À Lanhélin, pays de granite. Une exploitation de carrières ", Le Mausolée, 1969 (2), p. 323-334.

Chartier J.-J., 2006 - "Les carrières mont-doloises aux XIx ${ }^{\mathrm{e}}$ $\mathrm{xx}^{\mathrm{e}}$ siècles ", Le Rouget, $\mathrm{n}^{\circ} 89$, p. 24-35; n 90 , p. 39-51.

Chauris L., 1956 - «Sur les relations du Cambrien et du granite de Vire (Normandie) ", C.-R. de l'Académie des Sciences, 242, p. 3092-3094.

Chauris L., 2002 - «Les carrières exploitées pour la construction du viaduc de Dinan (1846-1852) ", Le Pays de Dinan, 22, p. 219-241.

Chauris L., 2005 - "Le granite de Languédias dans le pays de Dinan ", Bulletin de l'Association bretonne, 114, p. 191-208.

Chauris L., 2006 - "Le calcaire du Quiou-Tréfumel ou "pierre de jauge" ", Le Pays de Dinan, 116, p. 319-339.

Chauris L., 2009 - "Propos "terre à terre" sur le Mont-Dol ", Mémoires de la Société archéologique et historique d'Ille-etVilaine, 113, p. 27-33.

Chauris L., 2010 - "Impacts du granite de Bécherel (Ille-etVilaine) dans les édifices religieux ", Bulletin de la Société archéologique et historique d'Ille-et-Vilaine, 114, p. 27-40.

Chauris L., 2011 - "Saint-Malo : la pierre et la mer ", Mémoires de la Société d'histoire et d'archéologie de Bretagne, 89, p. 5-37.

Chauris L., (sous presse a) - «Sur la mise en œuvre pluriséculaire du granite de Saint-Broladre (Ille-et-Vilaine) ", Mémoires de la Société archéologique et historique d'Ille-et-Vilaine.

Chauris L., (sous presse b) - "La pierre de Dingé en Bretagne. Un granite oublié ", Bulletin du Musée de la Pierre, (Maffle, Belgique).

Chauris L., (sous presse c) - «En Bretagne, la pierre dans les ouvrages d'art du chemin de fer dans le département des Côtes-d'Armor et ses abords ", Pierre Actual.

Chauris L., Dangeard L., Graindor M.-J. et de Lapparent A.-F., 1956 - « Les principaux batholites granitiques du Bocage normand sont antérieurs à la transgression cambrienne ", $C .-R$. de l'Académie des Sciences, 243, p. 77-79.

Cochard J.-F., 2006 - "Alignement du Xxi ${ }^{\mathrm{e}}$ siècle. Une sculpture mégalithique d'Aurélie Nemours ", Pierre Actual, n 840, p. 76-79.

Collectif, 1998 - Roches de France, Ternay, Éditions Pro Roc, $226 \mathrm{p}$.

Cucarull J., 2001 - Le granit en Ille-et-Vilaine. Une économie, des hommes, un patrimoine, Rennes, Calligraphy Print, $88 \mathrm{p}$.

DU FAIL N., 1842 - Balivernes ou contes nouveaux d'Eutrapel, Paris, J.-M. Guichard.

GARGi R., 1971 - "Équipements modernes sur une carrière de Lanhélin " Le Mausolée, n 417, p. 1047-1055.

GARGi R., 1979 - « Le bassin d'extraction d'un grand granit français », Le Mausolée, n 514, p. 1087-1121.

Graindor M.-J., 1953 - "Sur l'âge du granite d'Athis (Orne) ", C.-R. de l'Académie des Sciences, 236, p. 504-506. 
Jonin M., 1969 - Étude pétrographique du massif granitique de Bonnemain (thèse de $3^{\mathrm{e}}$ cycle), Université de Clermont-Ferrand, $88 \mathrm{p}$.

JoNIN M., 1981 - Un batholite fini-précambrien, le batholite mancellien (Massif armoricain, France), thèse de doctorat-ès-Sciences naturelles, Brest : université de Bretagne occidentale, 338 p.

Joret E. (coord.), 2012 - L'Avenir. Chronique d'une coopérative granitière (1921-1985), Rennes, Archives départementales d'Ille-et-Vilaine, $160 \mathrm{p}$.

LAOUÉNAN J.-P., 1983 - "Les leucogranites de la marge nord de la Mancellia (Massif armoricain) dans leur cadre structural " (thèse de $3^{\mathrm{e}}$ cycle), Caen, Université de Caen - BasseNormandie, $192 \mathrm{p}$.

De Lapparent A., 1877 - « Sur le granite du Mont-Saint-Michel et sur l'âge du granite de Vire ", Bulletin de la Société géologique de France, 6, p. 143-147.

Leguay J.-P., 1980 - « Le paysage périurbain au Xve siècle; l'aspect et le rôle de la campagne voisine dans la vie de sociétés bretonnes au Moyen Âge ", Mémoires de la Société d'Histoire et d'Archéologie de Bretagne, 56, p. 63-127.
Leguay J.-P., 2001 - «Une ville épiscopale : Dol des origines au début du XvI ${ }^{\mathrm{e}}$ siècle ", Mémoires de la Société d'Histoire et d'Archéologie de Bretagne, 79, p. 5-73.

Mérimée P., 1836 - Notes d'un voyages dans l'Ouest de la France - réédition 2003 "Notes de voyage ", Paris, Société nouvelle Adam Biro, 214 p.

Monnier J.-L. et Molines N., 2003 - «Simon Sirodot et le site moustérien du Mont-Dol (Ille-et-Vilaine) : d'un grand précurseur aux recherches actuelles ", Bulletin de l'Association bretonne, $112\left(130^{\mathrm{e}}\right.$ congrès, Dol 2003, p. 55-88 [avec riche bibliographie].

Pierre M., 1962 - L'industrie du granit en Bretagne, Rennes, Centre régional d'études et de formation économiques, $115 \mathrm{p}$.

Pruvost P., 1949 - « Les mers et les terres de la Bretagne aux temps paléozoïques", Annales Hébert et Haug, 7, p. 345-362.

VAndernotte L., 1913 - Contribution à l'étude géologique des roches éruptives de la bordure nord-est du Massif armoricain (thèse de doctorat d'État), Paris, 191 p.

Zusammenfassung: Für eine Geoarchäologie des kulturellen Erbes: Gesteine, Steinbrïche und Gebäude in der Bretagne. 6. Teil: Cadomische Granite der Batholite des Mancellien - Der Osten der Bretagne wird ausschließlich von den granitischen Batholiten des Mancellien geprägt, den am weitesten westlich gelegenen intrusiven Tiefengesteinskörpern magmatischer Genese (Plutone), die am Ende des Präkambrium den Nordosten des amorikanischen Massivs formten. Diese Plutone weisen regelmäßig die gleichen Charakteristika auf. Die einen (Mont-Dol, Saint-Broladre, Dingé, Bécherel) wurden in früherer Zeit in erster Linie für lokale Zwecke ausgebeutet und genutzt; ihre Nutzung ist heute aufgegeben. Zwei andere, die heute immer noch abgebaut werden, haben eine nationale (Louvigné) oder sogar internationale Bedeutung (Lanhélin), die an dieser Stelle eine ausführliche Darstellung rechtfertigt.

Resumen: Para una geo-arqueología del patrimonio: piedras, yacimientos y construcciones en Bretaña. Sexta parte: los granitos cadomiens del batolito mancellien - El este de Bretaña no está concernido por los plutones más occidentales del gran batolito granitica mancellien implantados en el noreste del Macizo armoricano a finales del Precámbrico. Con frecuencia tienen cierta similitud. Algunos (Mont-Dol, Saint-Broladre, Dingé, Bécherel) sirvieron esencialmente para un uso local y ahora están abandonados. Otros dos, todavía explotados, tienen una difusión nacional (Louvigné) e incluso internacional (Lanhélin), lo que justifica amplio desarollo.

Schlüsselwörter: Mancellia, Granite, Abbau, Nutzung, Louvigné, Lanhélin.

Palabras clave: Mancellia, granitos, extracciones, usos, Louvigné, Lanhélin. 